Article

\title{
Expression of EMT-Related Genes in Hybrid E/M Colorectal Cancer Cells Determines Fibroblast Activation and Collagen Remodeling
}

\author{
Irina Druzhkova ${ }^{1}$, Marina Shirmanova ${ }^{1}, \operatorname{Nadezhda~Ignatova~}^{1}\left(\mathbb{D}, \operatorname{Varvara} \operatorname{Dudenkova}^{1}(\mathbb{D})\right.$ \\ Maria Lukina ${ }^{1}$, Elena Zagaynova ${ }^{1,2}{ }^{\text {, Dina Safina }}{ }^{3}$, Sergey Kostrov ${ }^{3}$, Dmitry Didych ${ }^{4}$, \\ Alexey Kuzmich ${ }^{3,4, *(\mathbb{D})}$, George Sharonov ${ }^{1,4,5}{ }^{\mathbb{D}}$, Olga Rakitina ${ }^{4}\left(\mathbb{D}\right.$, Irina Alekseenko ${ }^{3,4,6}$ \\ and Eugene Sverdlov ${ }^{3,7}$
}

1 Research Institute of Experimental Oncology and Biomedical Technologies, Privolzhsky Research Medical University, 603005 Nizhny Novgorod, Russia; danirin@yandex.ru (I.D.); shirmanovam@gmail.com (M.S.); n.i.evteeva@gmail.com (N.I.); orannge@mail.ru (V.D.); kuznetsova.m.m@yandex.ru (M.L.); ezagaynova@gmail.com (E.Z.); sharonov@gmail.com (G.S.)

2 Lobachevsky State University of Nizhny Novgorod, 603950 Nizhny Novgorod, Russia

3 Department of Molecular-Genetic Basis of Biotechnology and Protein Engineering, Institute of Molecular Genetics of National Research Centre «Kurchatov Institute», 123182 Moscow, Russia; nauruz@mail.ru (D.S.); kostrov@img.ras.ru (S.K.); irina.alekseenko@mail.ru (I.A.); edsverd@gmail.com (E.S.)

4 Department of Genomics and Postgenomic Technologies, Shemyakin-Ovchinnikov Institute of Bioorganic Chemistry of The Russian Academy of Sciences, 117997 Moscow, Russia; dmitrydid@gmail.com (D.D.); rakitinaolga97@gmail.com (O.R.)

5 Institute of Translational Medicine, Pirogov Russian National Research Medical University, 117997 Moscow, Russia

6 Laboratory of Epigenetics, FSBI «National Medical Research Center for Obstetrics, Gynecology and Perinatology named after Academician V.I. Kulakov» Ministry of Healthcare of the Russian Federation, 117198 Moscow, Russia

7 National Research Center «Kurchatov Institute», 123182 Moscow, Russia

* Correspondence: akrubik@gmail.com

Received: 28 September 2020; Accepted: 27 October 2020; Published: 30 October 2020 updates

\begin{abstract}
Collagen, the main non-cellular component of the extracellular matrix (ECM), is profoundly reorganized during tumorigenesis and has a strong impact on tumor behavior. The main source of collagen in tumors is cancer-associated fibroblasts. Cancer cells can also participate in the synthesis of ECM; however, the contribution of both types of cells to collagen rearrangements during the tumor progression is far from being clear. Here, we investigated the processes of collagen biosynthesis and remodeling in parallel with the transcriptome changes during cancer cells and fibroblasts interactions. Combining immunofluorescence, RNA sequencing, and second harmonic generation microscopy, we have explored the relationships between the ratio of epithelial (E) and mesenchymal (M) components of hybrid E/M cancer cells, their ability to activate fibroblasts, and the contributions of both cell types to collagen remodeling. To this end, we studied (i) co-cultures of colorectal cancer cells and normal fibroblasts in a collagen matrix, (ii) patient-derived cancer-associated fibroblasts, and (iii) mouse xenograft models. We found that the activation of normal fibroblasts that form dense collagen networks consisting of large, highly oriented fibers depends on the difference in E/M ratio in the cancer cells. The more-epithelial cells activate the fibroblasts more strongly, which correlates with a dense and highly ordered collagen structure in tumors in vivo. The more-mesenchymal cells activate the fibroblasts to a lesser degree; on the other hand, this cell line has a higher innate collagen remodeling capacity. Normal fibroblasts activated by cancer cells contribute to the organization of the extracellular matrix in a way that is favorable for migratory potency. At the same time, in co-culture with epithelial cancer cells, the contribution of fibroblasts to the reorganization of ECM is more
\end{abstract}


pronounced. Therefore, one can expect that targeting the ability of epithelial cancer cells to activate normal fibroblasts may provide a new anticancer therapeutic strategy.

Keywords: cancer-associated fibroblasts; colorectal cancer cells; collagen remodeling; fibroblast activation; epithelial/mesenchymal state

\section{Introduction}

It is known that the development and progression of malignant tumors, as well as their therapeutic resistance, depends not only on the intrinsic characteristics of the cancer cells but also to a significant degree on the tumor stroma [1,2]. A better understanding of the tumor-stroma interaction mechanisms is important in respect of the search for novel prognostic markers and therapeutic targets. The majority of experimental data demonstrate the improvements of therapeutic outcomes due to the application of stroma-targeting approaches, especially combined with traditional therapies [3]. However, there are no completely curative strategies and some of them, particularly in case of lysyl oxidase homolog 2 LOXL2 inhibitors, have failed clinical trials [3,4]. Taken together, it says that our knowledge in this field is insufficient, and the search for novel stromal targets should be carried out.

Among the stromal components, cancer-associated fibroblasts (CAFs) supposedly play an important role in the formation of the specific tumor microenvironment. CAFs represent a population of activated fibroblasts within tumors that is similar, in many ways, to the myofibroblasts involved in wound healing. It is believed that most CAFs derive from the activation of local tissue-resident fibroblasts, although the conversion of several other cell types (e.g., adipocytes, pericytes, endothelial cells) into CAFs is also documented [5]. The key differences of CAFs from normal (quiescent, non-activated) fibroblasts are their enhanced proliferation and biosynthetic activity, such as the production of extracellular matrix (ECM) components, remodeling enzymes, cytokines, and growth factors. Determining the molecular markers for the identification of CAFs is still an ongoing area of investigation [6]. The expression of the most commonly used markers- $\alpha$-smooth muscle actin (aSMA), fibroblast-activating protein (FAP), and platelet-derived growth factor receptors A/B (PDGFRa/b) - varies strongly among CAF subpopulations, reflecting the highly heterogeneous nature and plasticity of CAFs.

The exact mechanisms by which fibroblasts become activated in a tumor remain largely unknown. In the case of wound repair, the activation of fibroblasts is initiated by chemical mediators released by the damaged epithelial cells and by the immune cells recruited to the damage site. With respect to CAFs, the activation can be caused by additional chemical mediators produced by the cancer cells.

In a tumor, there is permanent crosstalk between the cancer cells and fibroblasts in which the cancer cells induce and support the activated phenotype of the fibroblasts, while the fibroblasts produce and remodel the ECM and stimulate the migratory activity of the cancer cells. As one of the results of such cooperation, the cancer cells acquire mesenchymal features, which can then contribute to their active migration and metastasis. This acquisition is associated with changes in the expression of genes involved in both intracellular and extracellular processes. In particular, the composition and presentation of surface adhesion molecules might change, as well as the activity of extracellular matrix reorganization and structuring processes.

Collagens are the major proteins of the ECM. In solid tumors, collagen is often cross-linked and linearized, which increases the tissue stiffness and provides paths for directed cancer cell invasion [7]. Previously, the desmoplastic stromal reaction characterized by the deposition of fibrillar collagens (primarily types I and III) and fibroblasts around the tumor was considered to be a response of the host tissue to the presence of invasive tumor cells, forming a barrier against the expansion of the cancer cells [8]. Recent studies suggest that desmoplasia, on the contrary, forms favorable conditions for tumor progression and metastasis via modulation of the biological behavior of the tumor cells, including their gene expression, proliferative activity, adhesion, migration, and apoptosis [9-11]. 
It has been shown that alterations in the microenvironment induced by CAFs (e.g., matrix stiffness) can initiate the phenotypic transformation of cancer cells from an epithelial to a mesenchymal state (the epithelial-mesenchymal transition, EMT). Nevertheless, the intrinsic epithelial/mesenchymal states of the cancer cells still remain an important determinant of the tumor-stroma co-evolution. The exact role of each of these components is still to be determined.

Another unanswered question is the role of cancer cells in organizing the collagen. Collagen synthesis and remodeling is classically regarded as one of the basic functions of activated fibroblasts in both normal and cancerous tissues. However, there are data showing that cancer cells, themselves, are capable of promoting contraction, cross-linking, and degrading collagen [12].

By now, the participation of the stroma in the tumor progression is most extensively investigated for breast cancer [13-16]. As for colorectal cancer, which is the third most common cancer type, there are only scattered data about the specifics of stroma organization [7,9-11]. The majority of the studies are focused on the regulation of the disease though the CAFs signaling, while the ECM is much less explored $[17,18]$. In this study, we sought to elucidate the correlations between the epithelial/mesenchymal states of colorectal cancer cells and their ability to activate normal fibroblasts and ECM remodeling. To this end, we exploited human colorectal cancer cell lines with different epithelial/mesenchymal states co-cultured with normal fibroblasts in a 3D collagen matrix. Patient-derived CAFs and colon tumor xenografts in mice were used to validate the in vitro findings. Using immunofluorescence assay, RNA-sequencing, and second harmonic generation (SHG) microscopy, we demonstrated phenotypic, transcriptomic, and functional changes in the fibroblasts upon their activation by cancer cells, and this correlated with the intrinsic migratory capacity of the cancer cells.

\section{Results}

\subsection{Epithelial/Mesenchymal States of Cancer Cells}

In a search for the cellular mechanisms underlying the interaction of cancer cells and normal fibroblasts, we examined various colorectal cancer cell lines for their epithelial/mesenchymal states. The well-described model cell lines HT29, HCT116, SW480, Caco-2, and SW837 were chosen for our experiments based on their ATCC description as cell lines with different epithelial/mesenchymal morphologies.

The wound-healing assay showed that the HT29 and SW837 cell lines had the least migratory activity (Figure 1A). Caco-2 healed wounds more quickly, but the process was not completed by $72 \mathrm{~h}$. HCT116 and SW480 showed an enhanced rate of wound closure compared with the other three cell lines.

It is known that the loss of ability to form spheroids correlates with increased migration and invasive activity of the cells [19]; therefore, we analyzed the cell lines for spheroid formation capacity (Figure 1B). It was found that two cell lines (SW480 and Caco-2) did not form cell aggregates by 7 days after seeding into ultra-low attachment round-bottom plates. The other three cell lines formed compact multicellular spheroids, but in the cases of HCT116 and SW837, a small proportion of the cells did not integrate into the spheres and was visible at the border.

Vimentin expression, loss of E-cadherin, enhanced migratory activity, ECM production, and invasiveness accompany the acquisition of mesenchymal features by cancer cells. We assessed the expression of E-cadherin and vimentin, which are two key markers of the epithelial-to-mesenchymal transition (EMT), using immunofluorescence staining. The E-cadherin level was the highest in HT29 cells and lowest in HCT116. The SW480, Caco-2, and SW837 cell lines displayed a moderate expression. Vimentin expression was detected only in the SW480 cells.

These data characterize HT29 as epithelial cells with a low level of migration activity and SW480 as the line with the highest migratory capacity and a mesenchymal phenotype. 
A

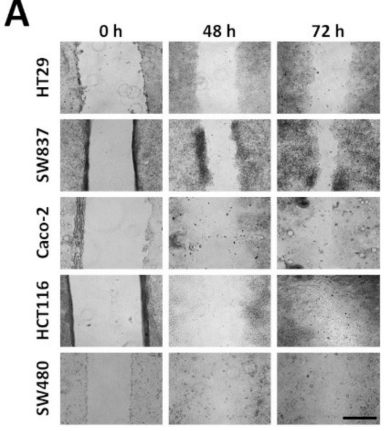

D

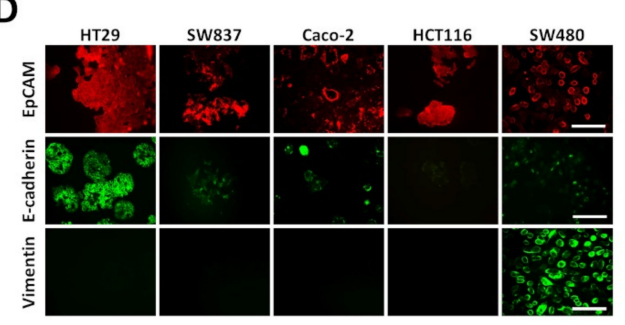

B

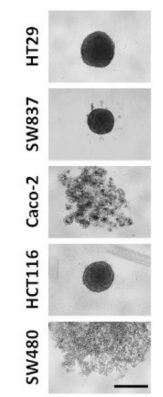

Mesenchymal cell markers

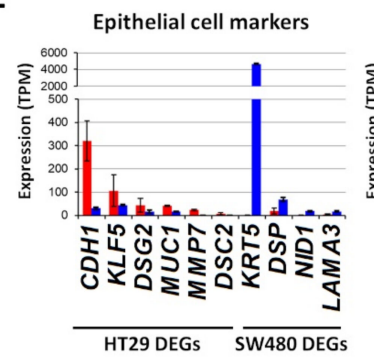

C

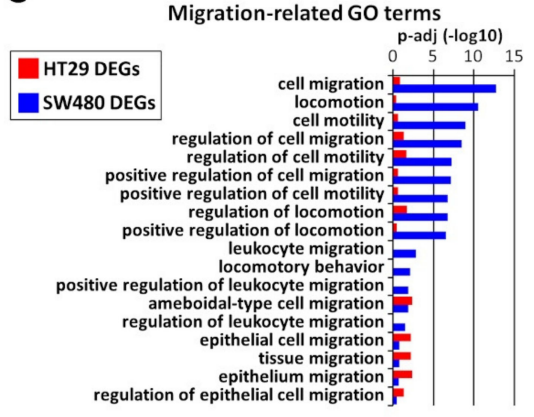

Adhesion-related GO terms

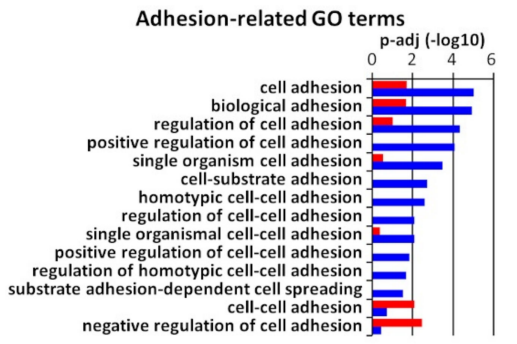
negative regulation of cell adhesion

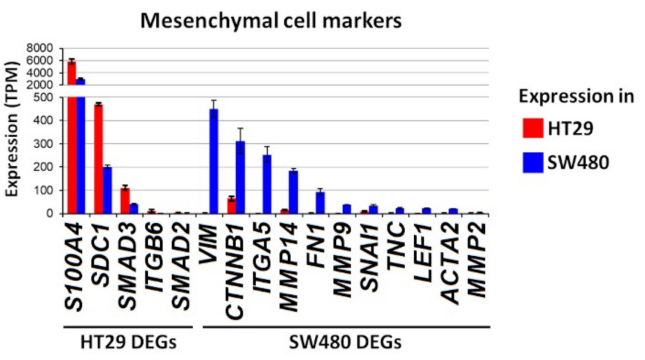

Figure 1. Identification of epithelial/mesenchymal state of five human colorectal cancer cell lines. (A) Monitoring of cell migration using wound-healing assay. Representative microscopic images of wound closure at 0,48 , or $72 \mathrm{~h}$ post wounding. Scale bar, $400 \mu \mathrm{m}$. (B) Spheroid formation ability of colorectal cancer cell lines. Cell lines were cultured on ultra-low attachment round-bottom plates, and the optical microscopy images were obtained 7 days after cell seeding. Scale bar, $400 \mu \mathrm{m}$. (C) Cell migration- and adhesion-related Gene Ontology Biological Process (GO BP) terms that are enriched in sets of differentially expressed genes between the HT29 and SW480 cancer cell lines. RNA-seq data were used to identify differentially expressed genes $(|\log 2(\mathrm{FC})|>1)$ that were subjected to functional enrichment analyses using the DAVID analysis tool to identify enriched GO BP terms. (D) Immunofluorescence staining of the indicated cell lines for the epithelial cell adhesion molecule (EpCAM) (red) and the epithelial marker E-cadherin (green) and mesenchymal markers vimentin (green). Representative fluorescence microscopic images showing the epithelial phenotype of HT29 cells and the mesenchymal phenotype of SW480 cells. Scale bar, $400 \mu \mathrm{m}$. (E) Expression of epithelial and mesenchymal marker genes differentially expressed between HT29 and SW480 cancer cells. RNA-seq data were used to calculate the gene expression levels in transcripts per kilobase million (TPM). Mean values \pm standard deviation in triplicate are shown.

To investigate the molecular mechanisms underlying the observed difference in the epithelial/mesenchymal states of the HT29 and SW480 cell lines, RNA-seq and subsequent transcriptomic comparisons were performed. The analysis (Supplementary Figure S1A) revealed 2044 differentially expressed genes (DEGs) in HT29 cells (that is, genes with $p$-adj <0.05; TPM (in HT29) $\geq 5$ and $\log 2$ (fold change, FC) $\leq-1$ ) and 2152 SW480 DEGs (that is, genes with $p$-adj $<0.05 ;$ TPM (in SW480) $\geq 5$ and $\log 2(\mathrm{FC}) \geq 1$ ). Among these genes, Gene Ontology-based functional enrichment analysis revealed 248 and 389 DEGs involved in cell migration and adhesion in the HT29 and SW480 lines, respectively (Supplementary Figure S1B). Cell migration and adhesion are known to be closely related processes [20]. We compared the Gene Ontology Biological Process (GO BP) terms related to cell migration and 
adhesion, for which significant $(p-a d j<0.05)$ enrichment was found in the studied HT29 and SW480 DEGs. In general, the enrichment in categories related to migration and adhesion is more typical for the SW480 DEGs (Figure 1C), thus confirming the observed phenotypic difference of these cell lines (Figure 1A,B). HT29 DEGs are enriched in only a few categories, those related to epithelial cell migration and collective migration ("tissue migration"), as shown in Figure 1C. These observations correspond to the observed increased mobility and invasiveness of SW480 cells compared to HT29 cells (Figure 1A,B).

Despite the greater activity of their cell-adhesion-related genes, SW480 cells were not able to form spheroids, in contrast to HT29 cells. This phenomenon might be explained by the low expression level in SW480 cells of the E-cadherin gene (CDH1), which is one of the key cell-contact and spheroid-formationrelated proteins, $[19,21]$.

We have also studied epithelial and mesenchymal marker genes [22-25], which were differentially expressed in HT29 and SW480 (Supplementary Tables S2 and S3, respectively). Among ten differentially expressed epithelial markers, four genes have higher expression in SW480 and six genes, including CDH1 and KLF5, have higher expression in HT29 cells (Figure 1E (left)). In addition, 16 differentially expressed mesenchymal marker genes were identified, 11 of which have higher expression in SW480 (including ITGA5, VIM, CTNNB1, MMP14, FN1, MMP9, and SNAI1), and five have higher expression in HT29 cells (Figure 1E (right)). The expression of EPCAM was similar in HT29 (237 TPM) and SW480 (255 TPM) cells, which corresponds well with the data from immunofluorescent staining (Figure 1D). The observed combination of epithelial and mesenchymal features in the SW480 cells demonstrates their hybrid phenotype, which is significantly biased to the mesenchymal, when compared to HT29 cells, thus confirming the difference between these cell lines as observed with immunofluorescent staining (Figure 1D).

Thus, the ICH staining, the transcriptome investigation, and the functional tests all confirmed the epithelial phenotype with low migratory capacity of the HT29 cell line and the mesenchymal phenotype with high migratory activity of the SW480 cell line.

\subsection{The Molecular Phenotype of NFs and CAFs and Activation of Normal Fibroblasts in Co-Culture}

We performed immunofluorescence analysis for two major CAF markers: FAP and aSMA in normal fibroblasts (NFs) and CAFs, which were isolated from the patients' colorectal tumors. Despite the FAP being considered as a CAF marker, in our experiments, the FAP expression in CAFs and NFs was nearly the same, while aSMA, which is typical of myofibroblasts, had a significantly higher expression level in the CAFs (Figure 2).

RNA-seqs of the NFs and two cultures of CAFs (CAF1 and CAF2) were performed to confirm the observed difference. Comparison of the expressions of 24 CAF marker genes (Supplementary Table S4; [26-33]) in the NFs with those in CAF1 and CAF2 revealed six genes, whose expression in CAF1 and CAF2 was considerably ( $>2$ times) higher than in the NFs (ACTA2, ASPN, CXCL10, $P D P N, T H Y 1$, and TNC). In addition, the POSTN expression level was more than 2-fold higher in CAF1 than that in the NFs ( $p$-adj $<0.05$; Figure 2E), and also, the TPM for this gene was $>5$ in CAF1. Thus, despite FAP expression being similar in the NFs and CAFs (Figure 2E), the activity of other known CAF markers was significantly higher in both isolated CAF lines compared to that in the NFs.

We simulated in vitro the NFs' interactions with cancer cells by the co-cultivation of NFs with the cancer cell lines, HT29, HCT116, and SW480, of which HT29 was preliminarily identified as the least invasive, and SW480 was preliminarily identified as the most invasive cell line.

With immunofluorescence assay, an elevated FAP expression was observed in the NFs of all three co-culture systems, starting at day 2 of culturing, with the maximum being in co-culture NF+HCT116. After that, in the case of NF+HCT116 and NF+SW480 the co-cultures, FAP expression dropped. In the case of NF+HT29 co-culture, the maximum FAP expression was achieved at day 5, which was followed by a slight decrease by day 7. A similar dynamic was observed for aSMA expression with a sharp increase by day 2, which was followed by gradual decrease by day 5 in all three co-cultures and a 
dramatic drop to the level registered in NFs in the NF+HCT116 and NF+SW480 co-cultures by day 7 . At day 1 in NF+SW480 co-culture, the aSMA expression was even lower than in the NFs, but it was accompanied by the maximal FAP expression for this co-culture. These data demonstrate the activation of normal fibroblasts in the presence of cancer cells, which is clearly seen from the expression of aSMA. An increase in FAP expression in the co-cultures was also observed, although normal skin fibroblasts originally had quite a high level of FAP, which is consistent with their physiological functions.
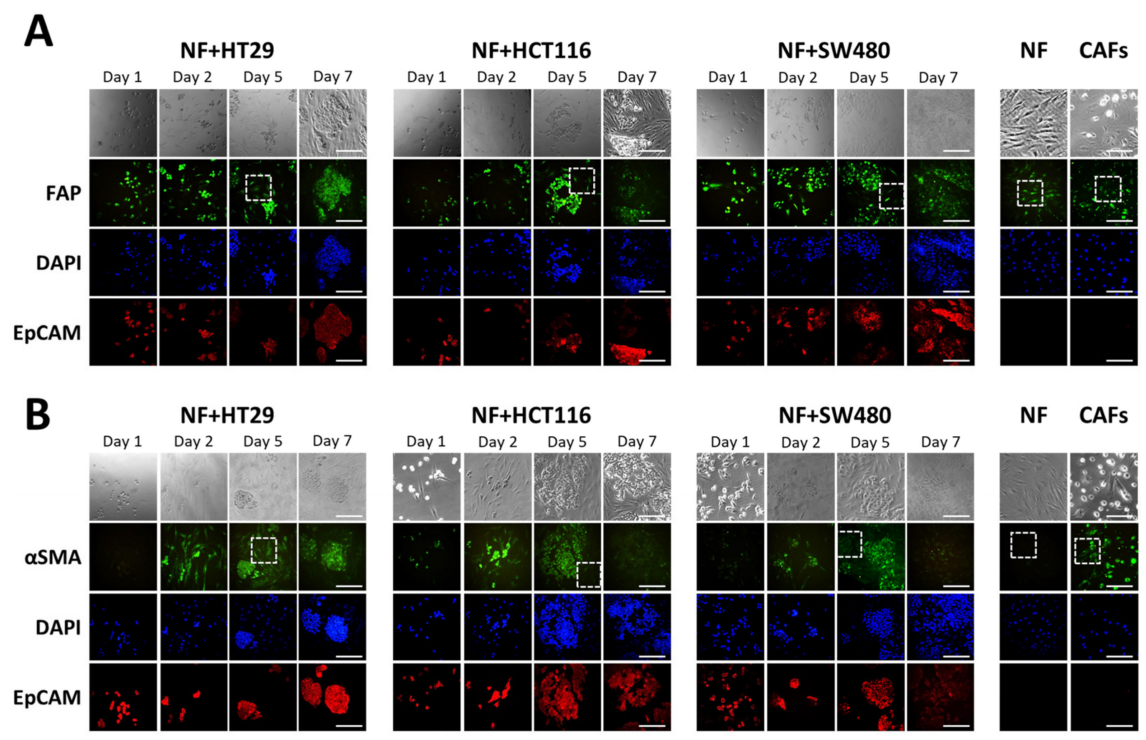

NF CAFs

C

D
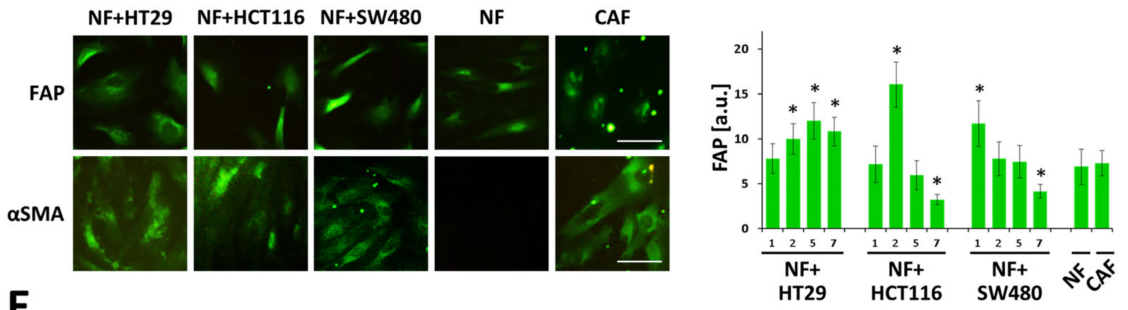

$\mathbf{E}$
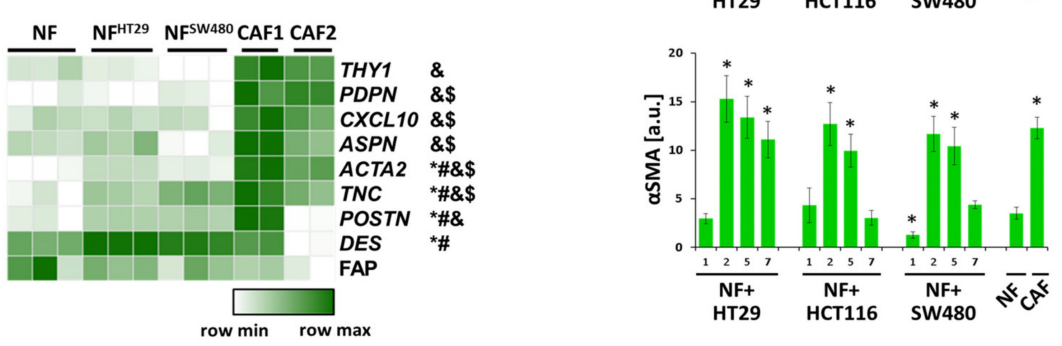

Figure 2. Activation of normal fibroblasts upon co-culturing with colorectal cancer cells. Immunofluorescence staining of the co-cultures of HT29, HCT116, or SW480 cells with normal fibroblasts (NFs) on days 1, 2, 5 and 7, NFs and patient-derived CAFs for (A) FAP, fibroblast activation protein $\alpha 1$, and (B) aSMA, $\alpha$-smooth muscle actin. Cells were additionally stained against 4',6-diamidino-2-phenylindole (DAPI; nucleus; blue) and EpCAM (epithelial cell adhesion molecule; red). Scale bar, $400 \mu \mathrm{m}$ (C) A higher magnification of the areas corresponding to the dashed square in (A), (B). Scale bar, $100 \mu \mathrm{m}$. (D) Quantification of FAP and aSMA staining (averaged fluorescence intensity of fibroblasts, at least eight regions of interest (ROIs) per condition with 2-5 cells in each ROI). Mean \pm SD. $*, p<0.05$ from NF. (E) Gene expression heatmap of cancer-associated fibroblast (CAF) markers with differential activity in various types of fibroblasts. NFs-normal skin fibroblasts, $\mathrm{NF}^{\mathrm{HT} 29}$-normal fibroblasts isolated from co-cultures with $\mathrm{HT} 29$ cancer cells on day 5, NF ${ }^{\mathrm{SW} 480}$ —normal fibroblasts isolated from co-cultures with SW480 cancer cells on day 5, CAF1-2 - colon cancer-associated fibroblasts (cultures 1 and 2). $p$-adj < 0.05 for gene expression in NFs compared to *, $\mathrm{NF}^{\mathrm{HT} 29}$; \#, $\mathrm{NF}^{\mathrm{SW} 480}$; \&, CAF1; \$, CAF2. 
It is notable that cancer cells in the co-cultures expressed high levels of FAP and aSMA, but their further analysis was out of the scope of this study.

Transcriptome analysis of NFs before and after 5 days of co-cultivation with the HT2 $\left(\mathrm{NF}^{\mathrm{HT} 29}\right)$ and $\mathrm{SW} 480\left(\mathrm{NF}^{\mathrm{SW} 480}\right)$ cell lines was performed to confirm the observed fibroblast activation. We compared the expression of the seven CAF marker genes mentioned above (ACTA2, ASPN, CXCL10, PDPN, THY1, TNC, and POSTN) in these cells and revealed a statistically significant (about 2-fold, $p$-adj $<0.05$ ) increase in ACTA2, POSTN, and TNC gene expression as a result of co-cultivation with both HT29 and SW480 cells (Figure 2E). The expression level of all three genes in both $\mathrm{NF}^{\mathrm{HT} 29}$ and $\mathrm{NF}^{\mathrm{SW} 480}$ was significantly lower than that in CAFs. We also found that the DES gene (one of the $24 \mathrm{CAF}$ marker genes) expression level was more than 2-fold higher in both $\mathrm{NF}^{\mathrm{HT} 29}$ and $\mathrm{NF}^{\mathrm{SW} 480}$ compared to NFs alone, its expression level being similar in CAFs and NFs in pure culture. The activation of the expression of ACTA2 (2.1-fold in $\mathrm{NF}^{\mathrm{HT} 29}$ vs. 1.3-fold in $\mathrm{NF}^{\mathrm{SW} 480}$ ) and DES (4.9-fold in $\mathrm{NF}^{\mathrm{HT} 29}$ vs. 3.4-fold in $\mathrm{NF}^{\mathrm{SW} 480}$ ) was more significant in $\mathrm{NF}^{\mathrm{HT} 29}$, the expression of POSTN was similarly activated in $\mathrm{NF}^{\mathrm{HT} 29}$ (1.6-fold) and NF ${ }^{\mathrm{SW} 480}$ (1.7-fold), and the activation of the expression of TNC (1.6-fold in $\mathrm{NF}^{\mathrm{HT} 29}$ vs. 2.2-fold in $\mathrm{NF}^{\mathrm{SW} 480}$ ) was more significant in $\mathrm{NF}^{\mathrm{SW} 480}$. Thus, the RNA-seq data might indicate that during co-cultivation, HT29 cells activate normal fibroblasts to a greater extent than do SW480s, which corresponds well with the immunofluorescence staining data. The ACTA2 expression enhancement as a result of co-cultivation correlates well with the results of aSMA immunofluorescent staining (Figure 2C,D). In addition, RNA-seq confirmed the similarity of FAP expression in the $\mathrm{NF}$ and NF ${ }^{\mathrm{SW} 480}$ cells (Figure 2C-E). However, the RNA-seq data were not consistent with the observed immunofluorescence enhancement of FAP staining in $\mathrm{NF}^{\mathrm{HT} 29}$ after 5 days of co-cultivation (Figure 2C-E). This result might be explained by the observed decreases in FAP expression during co-cultivation (Figure 2C,D). The decreases might be caused by the suppression of $F A P$ expression by the 5th day of co-cultivation, leading to the similar detected levels of FAP transcript in NFs and $\mathrm{NF}^{\mathrm{HT} 29}$. Meanwhile, the amount of surface FAP in $\mathrm{NF}^{\mathrm{HT} 29}$ detected at this point by immunofluorescence staining had not yet dropped.

Therefore, co-cultivation of NFs with cancer cells resulted in a moderate activation of individual CAF gene markers that allows us to conclude that activated fibroblasts at the moment of recruitment by cancer cells represent a bridging form of myofibroblasts rather than mature CAFs.

\subsection{Collagen Remodeling}

\subsubsection{Collagen structure and Collagen-Related Gene Expression in Monocultures of Cancer Cells}

The ability of three colorectal cancer cell lines (HT29, HCT116 and SW480) to remodel collagen was studied in collagen-based 3D models using SHG microscopy. It was found that cancer cells, when cultured alone, differ in their capacity to remodel collagen (Figure 3A); all quantitative parameters of the SHG signal and $p$-values are given in Supplementary Table S5. Overall, in monocultures of cancer cells, fibrillar collagen was present in low quantity. An increase in the density of collagen was observed on day 5 of cultivation only for two invasive cell lines, HCT116 and SW480. Of the three cell lines, SW480 demonstrated the ability to create quite thick, oriented fibers starting from day 5 , which correlates with its more mesenchymal phenotype. This effect is consistent with data on the expression of the genes involved in collagen production and remodeling (see below).

Collagen-related gene expression was assessed in HT29 (epithelial phenotype) and SW480 (mesenchymal phenotype) cells using RNA-seq (Figure 4A,B). The expression of genes, related to collagen biosynthesis and positive regulation $(\mathrm{B}+\mathrm{PR})$; collagen fibril organization and $\mathrm{ECM}$ aggregation $(\mathrm{CFO}+\mathrm{EA})$; and collagen catabolism and negative regulation $(\mathrm{C}+\mathrm{NR})$ was estimated. The genes were included in the gene categories according to GO BP terms with manual correction and [34], and the gene lists are shown in the Supplementary Tables S8-S10, respectively. Venn diagrams and histograms were used to compare the genes significantly expressed in HT29 and SW480 ( $p$-adj < 0.05; TPM $\geq 5)$ and show that more genes, related to collagen biosynthesis and positive regulation, and to collagen catabolism 
and negative regulation, are active in cell line SW480 compared to HT29 (Figure 4A,B). In spite of the fact that more genes, related to collagen fibril organization and ECM aggregation, are active in cell line HT29 compared to SW480, two important collagen-remodeling genes, LOXL2 and LOXL3 (lysyl oxidases), are more active in SW480 compared to HT29 (Figure 4B). The observed differences may indicate that SW480 cells interact with collagen much more actively than do HT29, and this corresponds well with the SHG microscopy data (Figure 3A).

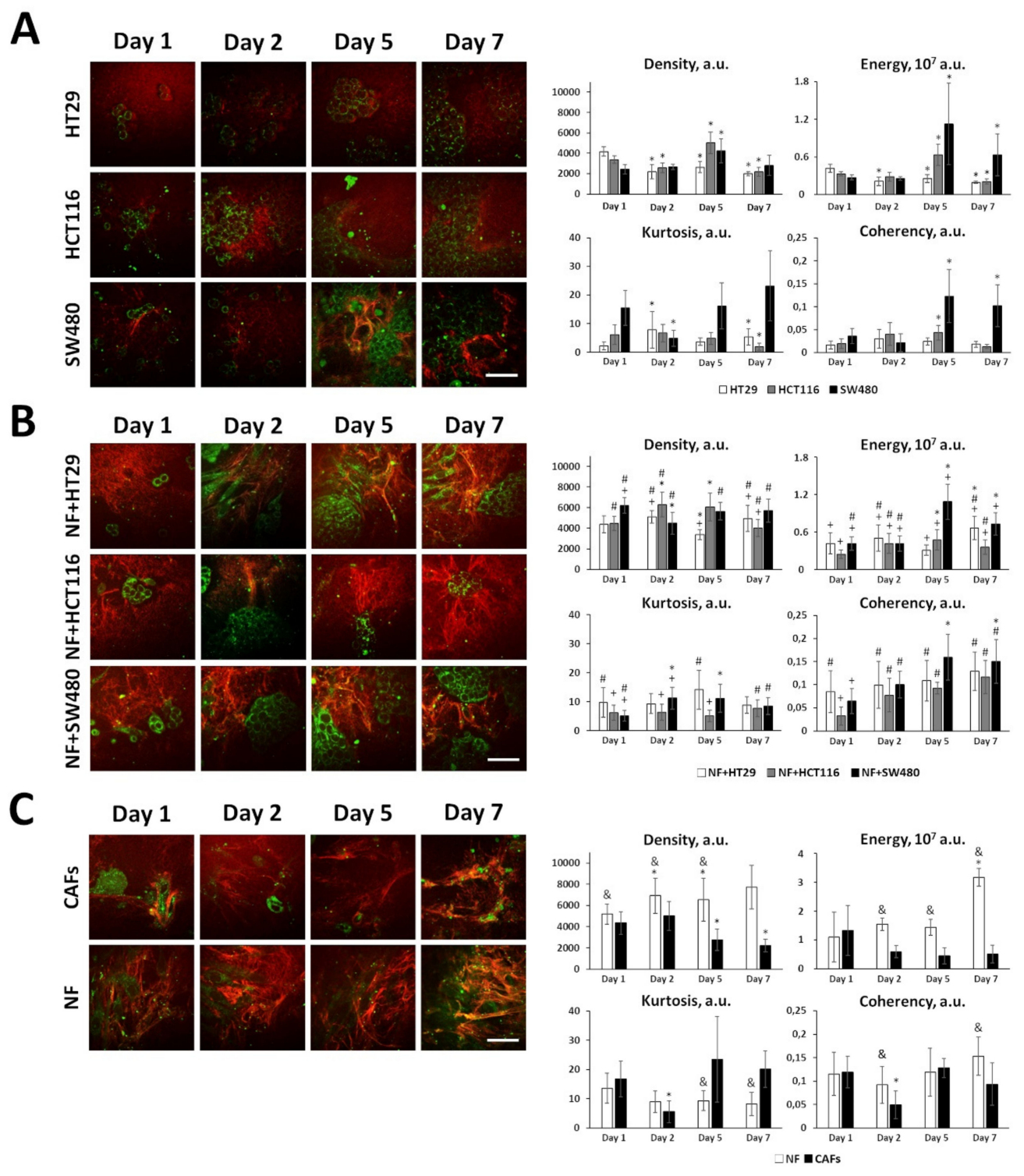

Figure 3. In vitro fibrillar collagen assessment in collagen-based 3D models using second harmonic generation (SHG) microscopy. The collagen structure in 3D systems containing cancer cells and/or fibroblasts in collagen gel was monitored with SHG microscopy and then quantified using the parameters of the SHG signal. Right: Representative SHG images (red) combined with two-photon excited fluorescence (TPEF, green) images of monocultures of cancer cells (A), co-cultures of cancer cells and normal fibroblasts (B), and monocultures of normal fibroblasts (NFs) and patient-derived CAFs (C). Bar is $50 \mu \mathrm{m}$, applicable to all images. Left: Several quantitative parameters of the SHG signals including density, energy, kurtosis, and coherency. Mean \pm SD. Imaging was performed at $1,2,5$, and 7 days of culturing cells in collagen gel. Key: ${ }^{*}, p<0.05$ from Day $1 ; \#, p<0.05$ from corresponding monoculture of cancer cells on the same day,,$+ p<0.05$ from NFs on the same day, $\&, p<0.05$ from CAFs on the same day. All values are given in arbitrary units (a.u.). All quantitative parameters of the SHG signal and $p$-values are given in Supplementary Tables S5-S7. 
A

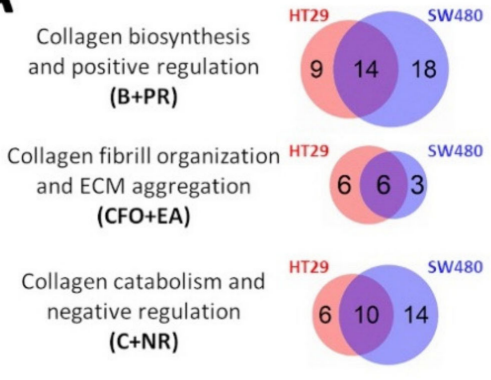

B

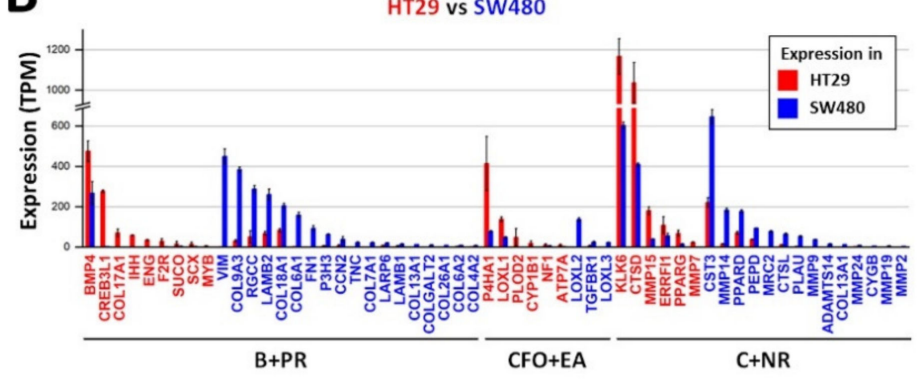

C
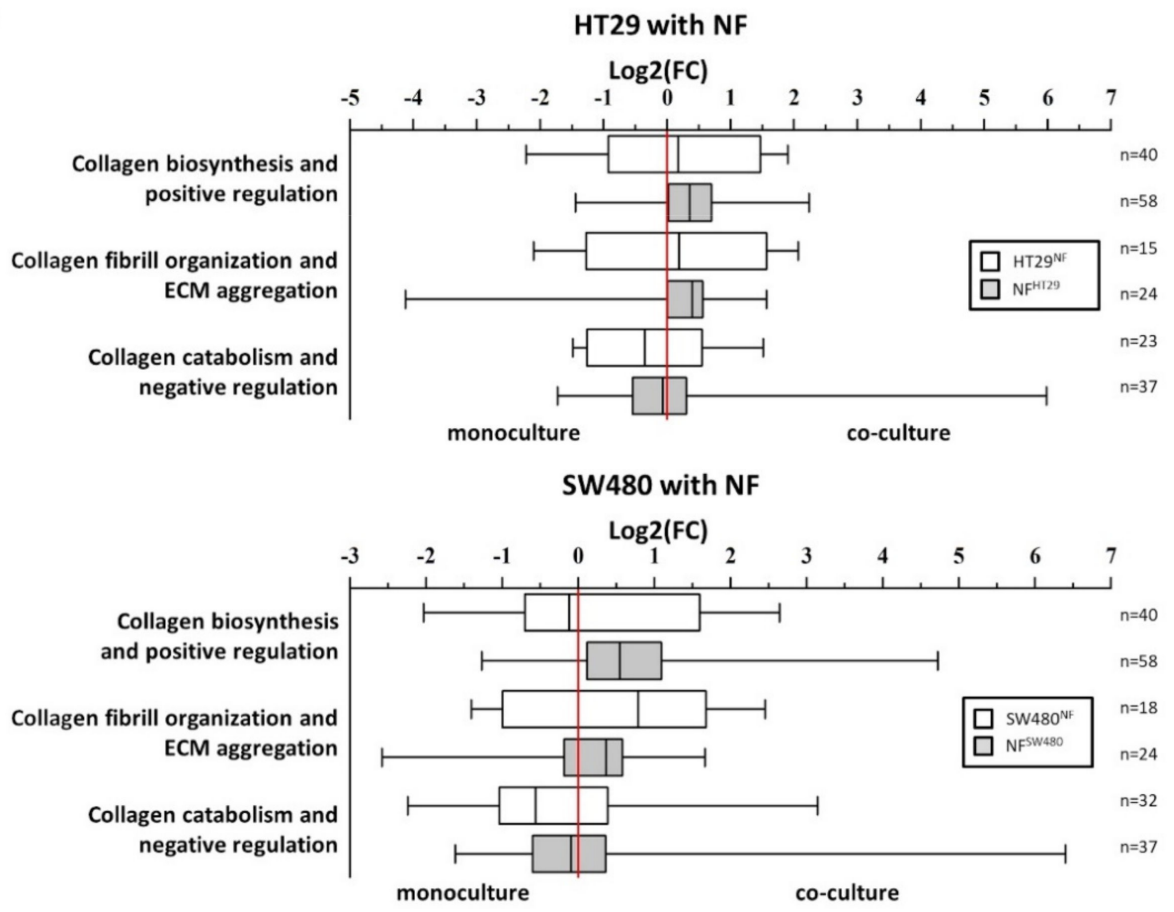

Figure 4. Expression of collagen-related genes in cancer cells and its changes as a result of the co-cultivation of cancer cells with normal fibroblasts. (A) Venn diagrams showing the overlap between significantly expressed collagen-related genes $(p$-adj $<0.05 ;$ TPM $\geq 5)$ in colorectal cancer cell lines HT29 and SW480 in monoculture. Three gene categories are shown: collagen biosynthesis and positive regulation $(\mathrm{B}+\mathrm{PR})$, collagen fibril organization and extracellular matrix $(\mathrm{ECM})$ aggregation $(\mathrm{CFO}+\mathrm{EA})$, and collagen catabolism and negative regulation $(\mathrm{C}+\mathrm{NR})$. (B) Histogram showing the expression of collagen-related genes, differentially expressed ( $p$-adj $<0.05 ; \mathrm{TPM} \geq 5 ;|\log 2(\mathrm{FC})| \geq 1$ ) in HT29 (red gene name) and SW480 (blue gene name). Red and blue bars represent the expression of genes in HT29 and SW480, respectively. Mean values $(n=3)$ are shown in TPM, error bars represent the standard deviation $(n=3)$. (C) Box plots representing changes of collagen-related gene expression as a result of co-cultivation of HT29 or SW480 cell lines with normal fibroblasts (NFs). The distributions of the $\log 2(\mathrm{FC})$ values for significantly expressed collagen-related genes $(p-a d j<0.05 ; \mathrm{TPM} \geq 5)$ in 3 gene categories $(\mathrm{B}+\mathrm{PR}, \mathrm{CFO}+\mathrm{EA}$, and $\mathrm{C}+\mathrm{NR})$ are shown; that is, the expression of the gene in co-culture divided by the expression of the gene in monoculture. Thus, gene expression that is up-regulated during co-cultivation has $\log 2(\mathrm{FC})>0$, and gene expression that is down-regulated during co-cultivation has $\log 2(\mathrm{FC})<0$. The twenty-fifth, fiftieth, and seventy-fifth percentiles are used; whiskers show the minimal and maximal log2(FC)-values. White boxes represent the changes of collagen-related gene expression in the cancer cells (HT29 or SW480); gray boxes represent the changes of collagen-related gene expression in the NFs. The red line indicates $\log 2(\mathrm{FC})=0$, that is, no change in gene expression. The number of genes $(n)$ used for each box is indicated on the right side of the chart. 
2.3.2. Collagen Remodeling and Collagen-Related Gene Expression in Co-Cultures of Cancer Cells and Normal Fibroblasts

In the presence of fibroblasts, the collagen structure significantly changed (Figure 3B); all quantitative parameters of the SHG signal and $p$-values for co-cultures are given in Supplementary Table S6. Co-culturing of all cancer cell lines with normal fibroblasts resulted in a statistically greater amount of fibrillar collagen during all periods of cultivation. Notably, the deposition of collagen was more pronounced in the case of the more invasive cells, HCT116 and SW480, where a 2-fold increase in the collagen density, compared with the monocultures, could be observed after 1-2 days of co-culturing. In the case of HT29, such an increase in the collagen density was achieved after 7 days. In the process of co-culturing, the fibers become more heterogeneous in size. Furthermore, a co-culture with HT29 formed an increased number of large fibers, as compared with the monocultures. The high proportion of thick fibers in the case of co-culture with SW480 may be attributed to the invasiveness of this cell line itself, rather than to its interaction with the fibroblasts. The most noticeable differences in the matrix architecture resulting from the interaction of cancer cells and fibroblasts were observed for the ordering of the collagen fibers. In all co-cultures, the fibers become more organized and more aligned with time, and particular orientations of the fibers could be distinguished. Note that such ordering of the fibers is a relatively early process during the interaction of the cancer cells and NFs; already after 1 day of co-culturing, the parameter of coherency exceeded that in the cancer cell monocultures and in pure collagen gel $(\approx 0.02 \mathrm{a}$.u).

Comparison of the collagen structures formed by NFs in monoculture (Figure 3C) and in the co-cultures revealed a greater collagen content and a more uniform structure of the collagen in the NFs monocultures. That is, the ECM in the NFs culture was represented by a large number of collagen fibers with equal size and uniform distribution, resulting in a dense homogeneous structure of the ECM, while in co-cultures, the ECM was less dense with non-uniformly distributed collagen fibers.

RNA-seq of HT29 and SW480 cancer cells and NFs before and after 5 days of co-cultivation was performed to compare the effects observed with the changes in gene expression patterns. The expression of collagen-related genes was estimated. Figure $4 \mathrm{C}$ shows the distributions of the $\log 2(\mathrm{FC})$ value for significantly expressed collagen-related genes in three gene categories $(\mathrm{B}+\mathrm{PR}, \mathrm{CFO}+\mathrm{EA}$, and $\mathrm{C}+\mathrm{NR})$; that is, the expression of the gene in co-culture divided by the expression of the gene in monoculture. The shift of the median of the $\log 2(\mathrm{FC})$ for genes related to collagen fibril organization and ECM aggregation toward co-cultured cancer cells (both HT29 and SW480) may indicate the activation of collagen remodeling processes in these cells as a result of the co-cultivation. Likewise, the shift of the median of the $\log 2$ (FC) for genes related to collagen catabolism and negative regulation toward cancer cells before co-cultivation (both HT29 and SW480) may indicate the suppression of collagen catabolic processes in these cells as a result of co-cultivation. The observed shifts of the median of log2(FC) for collagen biosynthesis and positive regulation genes category are too small to cause significant phenotypic effects.

In the case of fibroblasts, co-cultivation with both cancer cell lines tended to increase the expression of the genes related to collagen biosynthesis, collagen fibril organization, and ECM aggregation. In turn, the expression of genes related to collagen catabolism and negative regulation in the fibroblasts is slightly down-regulated. In general, the number of significantly expressed collagen-related genes was greater in the NFs compared to that in the cancer cells in both co-cultures (Figure 5A), which may indicate that NFs are the main collagen-remodeling component of the co-cultures.

To reveal differences between the co-cultures, we have also compared the significantly expressed collagen-related genes in co-cultivated cancer cells (HT29 ${ }^{\mathrm{NF}}$ vs. SW480 ${ }^{\mathrm{NF}}$ ) and fibroblasts $\left(\mathrm{NF}^{\mathrm{HT} 29}\right.$ vs. $\mathrm{NF}^{\mathrm{SW} 480}$ ), as shown in Figure 5. The main difference is observed between the cancer cells (Figure 5, Supplementary Figure S3), with the majority of differing genes being most evident between the HT29 and SW480 monocultures (Figure 4B). Despite the small number of collagen-related genes, which determine the difference between $\mathrm{NF}^{\mathrm{HT} 29}$ and $\mathrm{NF}^{\mathrm{SW} 480}$, it can be noted (Figure $5 \mathrm{~B}$, Supplementary Figure S3) that two genes from the collagen biosynthesis and positive regulation group, COL7A1 and 
COL5A3, were highly active in NFs monoculture, and they increased their activity only during co-cultivation with SW480. The MMP3 gene responsible for collagen proteolysis was also expressed at a high level in the NFs monoculture and down-regulated only during co-cultivation with SW480.

A

\section{Collagen biosynthesis and positive regulation}

\section{Collagen fibril organization and ECM aggregation}

\section{Collagen catabolism and negative regulation}

\section{HT29 ${ }^{\mathrm{NF}}$ vs SW480 NF}

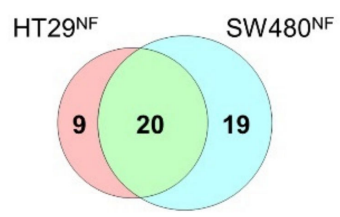

HT29NF

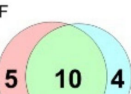

HT29NF

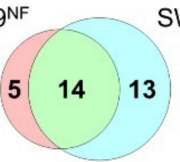

SW480NF

W480 NF

\section{(1)}

\section{$\mathrm{NF}^{\mathrm{HT} 29}$ vS NFSW480}
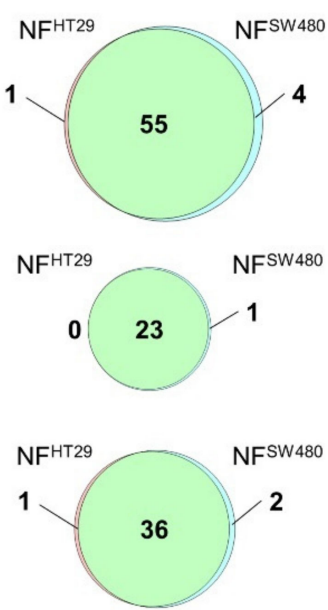

B

$\mathrm{NF}+\mathrm{HT} 29$ co-culture

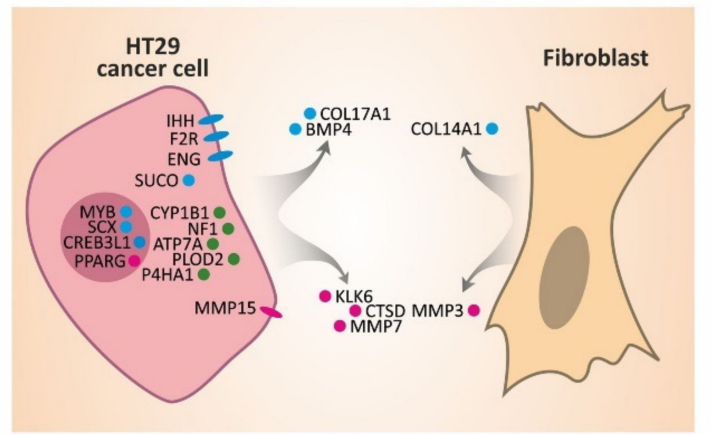

Collagen biosynthesis and positive regulation

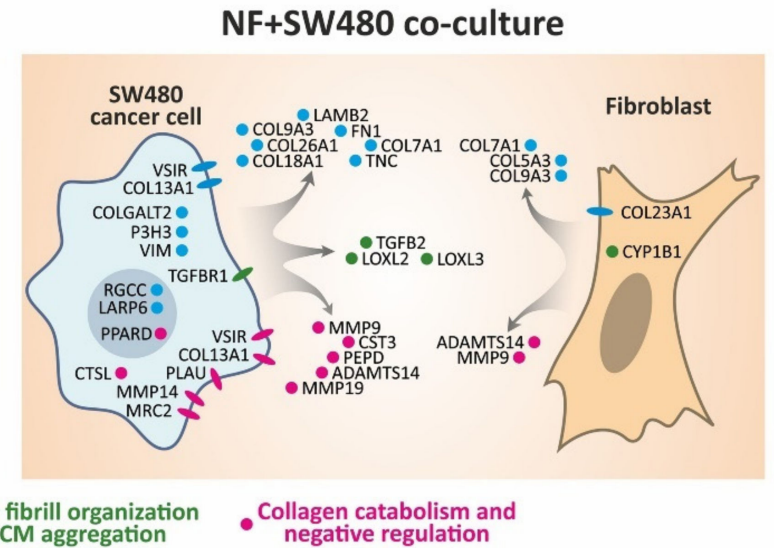

Figure 5. Differential expression of the collagen-related genes in co-cultivated cancer cells and fibroblasts. (A) Venn diagrams showing the overlap between significantly expressed collagen-related genes $\left(p\right.$-adj < 0.05; TPM $\geq 5$ ) in co-cultivated colorectal cancer cell lines $\left(\mathrm{HT} 29^{\mathrm{NF}}\right.$ and SW480 $\left.{ }^{\mathrm{NF}}\right)$ and normal fibroblasts $\left(\mathrm{NF}^{\mathrm{HT} 29}\right.$ and $\left.\mathrm{NF}^{\mathrm{SW} 480}\right)$. Three gene categories are shown: collagen biosynthesis and positive regulation, collagen fibril organization and ECM aggregation, and collagen catabolism and negative regulation. (B) Scheme showing localization of collagen-related proteins that can determine differences in collagen remodeling by different co-cultures. Collagen-related differentially expressed genes $(p$-adj $<0.05 ; \mathrm{TPM} \geq 5$; $|\log 2(\mathrm{FC})| \geq 1)$ ) were determined between cancer cells and between fibroblasts of both co-cultures. Some SW480 DEGs encoding extracellular proteins were excluded because of their much higher expression in fibroblasts $(\log 2(\mathrm{FC}) \geq 5)$.

Therefore, the observed up-regulation of the expression of the genes related to collagen fibril organization and ECM aggregation in both cancer cells and NFs and also the up-regulation of the expression of the genes related to collagen biosynthesis and positive regulation in NFs might explain the observed by SHG microscopy formation of the dense ECM, consisting of large, highly oriented collagen fibers (Figure 3B) as a result of co-cultivation. The observed difference in collagen remodeling between two co-cultures is most likely explained by the different collagen remodeling activities of the cancer cells present and not by any different collagen remodeling activity of the fibroblasts. 
2.3.3. Collagen Organization and Collagen-Related Gene Expression in CAFs and Normal Fibroblasts

A comparison of patient-derived CAFs and normal fibroblasts revealed strong differences in their ability to remodel collagen (Figure 3C); all quantitative parameters of the SHG signal and $p$-values for CAFs and NFs are given in Supplementary Table S7. Both types of cell culture formed dense collagen networks within 2 days; subsequently, however, in the culture of CAFs, the collagen density gradually decreased, while in the culture of normal fibroblasts, it did not change. In the culture of CAFs the number of collagen fibers was gradually reduced during cultivation, and only the larger collagen bundles were preserved, while in the culture of normal fibroblasts, a marked increase in the number and enlargement of collagen fibers were observed by day 7 . In both cases, the collagen was quite highly oriented (i.e., highly ordered), and the degree of ordering varied insignificantly during 7-day cultivation. Consequently, the major difference between CAFs and normal fibroblasts is a predominance of proteolytic processes in the CAFs culture, against which individual large collagen fibers are preserved, while normal fibroblasts tend to form a dense fibrous structure containing uniform fibers.

We have also compared the expression of three groups of collagen-related genes in NFs and CAFs (CAF1 and CAF2) (Supplementary Figure S2). The log2(FC) median of the B+PR gene group is slightly shifted toward normal fibroblasts compared to CAF1, which may indicate that collagen biosynthetic processes are more active in NFs than in CAF1.The other medians are not significantly shifted. These results support the SHG microscopy observations (Figure 3C), that is, a gradual increase in the number of collagen fibers during the cultivation of NFs, and proteolysis of collagen fibers during the cultivation of CAFs. Thus, compared to CAF1, normal fibroblasts might more actively participate in the processes of collagen biosynthesis.

Therefore, using SHG-based microscopy, we identified specific changes in the collagen morphology that develop when fibroblasts interact with cancer cells in vitro. Fibroblast-mediated remodeling of collagen leads to the formation of a highly ordered fiber network, which is more favorable for cancer cell invasion. Furthermore, the most invasive cancer cells, themselves, are capable of remodeling collagen to form oriented bundles and demonstrate the corresponding gene activity. Similar structural features of collagen were also typical of cultured CAFs isolated from patients' tumors. However, under prolonged cultivation in the absence of cancer cells, the CAFs lost the ability to organize collagen in ways to promote invasion and initiated matrix degradation. RNA-seq analysis demonstrated the elevated activity of all collagen-related genes in fibroblasts compared to cancer cells, confirming the predominant role of fibroblasts in collagen remodeling. Furthermore, the RNA-seq analysis, by revealing an increased activity of the collagen biosynthesis and organization genes in NFs compared to CAFs, suggests that NFs recruited by cancer cells are necessary participants in the tumor invasion front.

\subsubsection{Collagen Structure in Tumor Xenografts}

To determine if the innate epithelial/mesenchymal states of cancer cells influences collagen fiber organization in vivo, we inoculated HT29, HCT116, and SW480 cells subcutaneously into mice to generate tumors and assessed the resulting collagen properties using SHG microscopy.

Of these three cell lines, HT29 and HCT116 displayed high susceptibility (four of four tumors of each type) and similar growth rate in mice, reaching $\approx 700 \mathrm{~mm}^{3}$ in two weeks after inoculation. HT29 tumors had slightly slower growth rate in mice than HCT116 ( $\approx 180 \mathrm{~mm} 3$ vs. $\approx 400 \mathrm{~mm} 3)$ in two weeks after inoculation (Figure 6B). A SW480 tumor developed only in one of four animals and was much smaller in size, $\approx 50 \mathrm{~mm}^{3}$, and therefore, it was not considered. In spite of the different growth rate, histologically, both HT29 and HCT116 tumors had a dense, cellular structure with a moderate amount of stroma; the tumors contained a small number of necrotic cells, and the blood vessels were present mainly in the periphery (Figure 6A). 
A

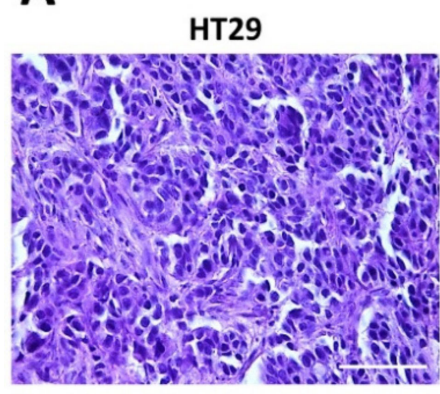

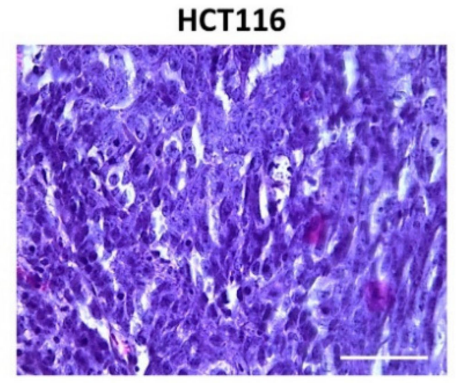

B

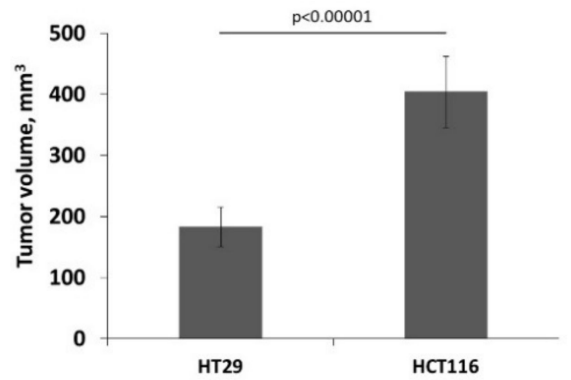

C

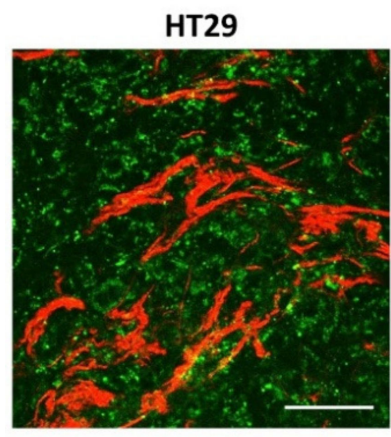

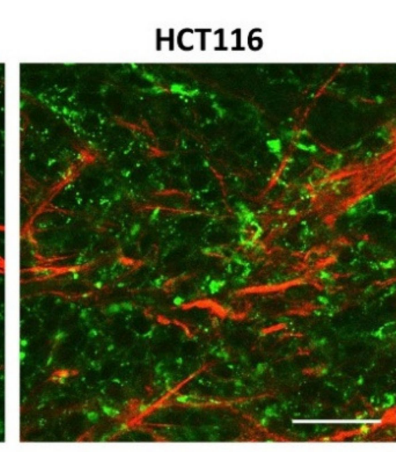

D

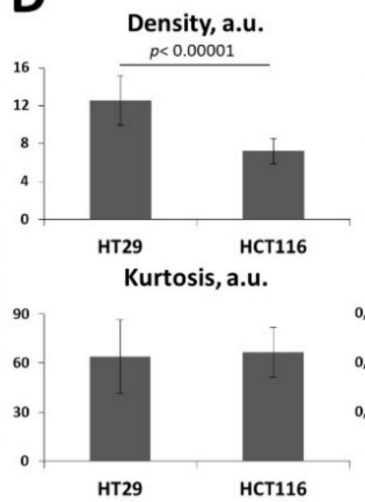

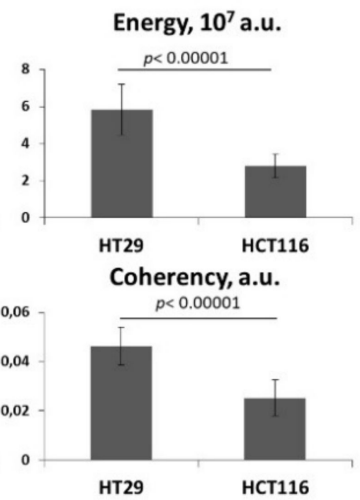

Figure 6. In vivo collagen structure in HT29 and HCT116 tumor xenograft. (A) Histopathology images on the 14th day of tumor growth. Hematoxylin and eosin staining. Scale bar, $50 \mu \mathrm{m}$. (B) Tumor volume (V) measurements on 14th day of growth. Mean \pm SEM. (C) Representative SHG images (red, collagen fibers) combined with two-photon excited fluorescence images (TPEF, green, cellular autofluorescence) of tumors. Image size is $212 \times 212 \mu \mathrm{m}$. Scale bar, $50 \mu \mathrm{m}$. (D) Quantitative parameters of the SHG signal including density, energy, kurtosis, and coherency. Mean \pm SEM, $(n=3-4$ tumors, at least 15 fields of view in each tumor). Cancer cells were implanted subcutaneously in nude mice. Imaging was performed on Day 14 of tumor growth. SHG images and quantitative analysis of the SHG signal show a denser and more oriented collagen structure in the HT29 compared with HCT116 tumors. $p$-values denote significant differences.

Therefore, a comparison of collagen structure in vivo was made for the HT29 and HCT116 tumors. We found that compared to HCT116, HT29 formed a denser stroma with more non-uniform and more oriented collagen fibers (Figure 6C,D). This observation was confirmed by the quantitative metrics. The parameters of the SHG signal, such as density, energy, and coherency showed statistically significant differences $(p<0.00001)$ between these tumor types.

The collagen organization in vivo in the HCT116 and HT29 xenografts results are consistent with the capacity of these two cell lines to activate normal fibroblasts, as was shown by immunofluorescence staining for FAP and aSMA in vitro. The activation of fibroblasts in the presence of HT29 cells was more excessive and prolonged than in the case of HCT116. This result allows us to suggest that cancer cells with a more-epithelial phenotype stimulate fibroblasts to a greater extent, ensuring a fibrous microenvironment.

\section{Discussion}

This work demonstrates the links between the epithelial/mesenchymal states of colorectal cancer cells, their ability to induce the transformation of normal fibroblasts into CAFs, together with the dynamics of this process and the organization of collagen. For the first time, communication between cancer cells and normal fibroblasts has been investigated at the molecular, phenotypic, and functional 
levels within one study. RNA profiling was performed to provide insight into the molecular mechanisms related to fibroblast activation and remodeling of the extracellular matrix.

\subsection{Communication of Colorectal Cancer Cells and Normal Fibroblasts Induces Activation of the Normal Fibroblasts}

Previously, numerous studies have demonstrated close interactions between different tumor components: cancer cells, the extracellular matrix, and non-cancerous cells associated with tumors, among which CAFs are the most abundant type of cells [35]. Usually, CAFs are described as activated cells with myofibroblast-like morphology distinguishable from that of resident tissue fibroblasts by their elevated production of cytokines, chemokines, metabolites, enzymes, and extracellular matrix components [36]. The precise mechanism of their emergence has not yet been determined [37], although resident quiescent fibroblasts are considered to be the main source from which CAFs originate $[36,38,39]$. Despite the abundant list of potential markers of fibroblast activation $[36,40]$, there are no unique characteristic for all CAFs. Markers can differ depending on tumor type and may dynamically change during cancer progression, likely reflecting the CAFs' plasticity [37].

Obtaining colon fibroblasts from healthy donors is arduous; therefore, to investigate the activation process of normal fibroblasts by cancer cells, we used skin fibroblasts from healthy donors. These reportedly, upon activation, have similar phenotypic features to myofibroblasts from skin wounds, those from pathological fibrotic tissue, and the fibroblasts in and around epithelial tumors [41]. It has been demonstrated that breast cancer cells activate normal dermal and mammalian fibroblasts in the same way [42], with the effects of dermal and colon fibroblasts on colon cancer cells being quite similar [43]. It has also been shown that HT29 cells activate normal colon fibroblasts in a way similar to our results in terms of aSMA expression and the elevation of ECM proteins [44]. In addition, the formation of highly ordered collagen structures is typical of all known pathological processes where myofibroblasts act as major participants $[45,46]$. So, we assume that skin fibroblasts can adequately model the processes typical of resident colon fibroblasts interacting with cancer cells in respect of ECM remodeling and cancer specific activation.

We used two major CAF markers-FAP and aSMA — to assess the dynamic changes in the NFs during co-cultivation with three colorectal cell lines. The activation of normal fibroblasts was clearly demonstrated by increases in the expression of FAP and aSMA at the beginning of their interaction with the cancer cells, although the expression of both markers decreased after further co-cultivation. Notably, the FAP expression demonstrated a sharp decline in co-cultures with the more-invasive cancer cells (HCT116 and SW480) but a gradual decrease in the presence of less-invasive cancer cells (HT29). In general, the aSMA expression had the same dynamics.

Data on the direct activation of fibroblasts by cancer cells are scarce, which is likely due to the absence of specific markers of activation. However, increases in FAP expression in human dermal fibroblasts and human primary mammary fibroblasts after treatment with conditioned medium from breast cancer cells [34] and elevated FAP expression in NFs after treatment with conditioned medium from the colorectal cancer cell line HCT116 [47] have been reported. The stable activated state of NFs typical of co-cultures of HT29+NFs in our study is consistent with the study by Peng et al., where the co-cultivation of NFs with HT29 cells resulted in the highest FAP and aSMA expression at Day 4 of co-cultivation among six cancer cell lines [48]. The long duration of the activated state of the NFs and their elevated expression of activation markers in our experiments correlate with higher expression of integrin $\alpha \mathrm{v} \beta 6$ (similar expression level to the ITGAV gene and 12.2-fold, $p$-adj $<0.05$ for the ITGB6 gene) and of E-cadherin (12.5-fold, $p$-adj < 0.05) in HT29 cells compared to SW480. The mechanistic relationship of integrin $\alpha \mathrm{v} \beta 6$ expression in cancer cells with the fibroblast activation was revealed in the study by Peng et al. [48]. HT29 cells secreted inactive transforming growth factor $\beta$ (TGF- $\beta$ ) and also expressed integrin $\alpha v \beta 6$, which subsequently activated the TGF- $\beta$. Then, the activated TGF- $\beta$ induced morphological changes in inactive fibroblasts and elevated the expression of activated fibroblast markers such as $\alpha$-smooth muscle actin ( $\alpha$-SMA) and fibroblast-activating protein (FAP). 
The association between the activation of normal human dermal fibroblasts and E-cadherin and EpCAM expression in cancer cells was also shown by Eberlein et al. [49] for different non-small cell lung cancer (NSCLC) cell lines. The connection between the elevated expression of E-cadherin and the activation of fibroblasts could be explained by the contribution of E-cadherin to cell-cell contacts, which could result in more effective transmembrane signaling.

It should be noted that immunohistochemical staining of colorectal and breast cancer specimens has demonstrated the elevated expression of FAP and aSMA mainly in the interstitial/border zone of tumors, while in the tumor core, the expression of these markers is less pronounced [47,50]. Our in vitro model reflects the process of recruitment of NFs into the tumor. This occurs at the tumor border, and therefore, it is rational that FAP expression in NFs in co-cultures was higher at the beginning of the interaction with cancer cells than that in tumor-derived CAFs, and that it decreased with time. Taken together, the results enable us to suggest that the transformation of NFs into CAFs is a dynamic process, which agrees with the concept of fibroblast plasticity [5,37]. The heterogeneity of CAFs has been confirmed by numerous studies; in particular, FAP or ACTA2 (aSMA) up-regulation was demonstrated not to occur for all CAF populations within a single tumor [51].

In our experiment with the use of RNA-seq analysis, the expression of podoplanin (PDPN), one of the CAF markers [38], was detected only in CAFs, but not in either NF monocultures or co-cultures of NFs with cancer cells. Recently, the PDPN expression in CAFs has been associated with poor prognosis for lung [52,53] and pancreatic cancers [54]. For colorectal cancer, it has been demonstrated that PDPN-positive CAF phenotype was associated with less aggressive tumors, whereas PDPN-low/ $\alpha$-SMAhigh or PDPN-low/S100A4high CAFs were associated with tumor progression [17]. The expression of several other CAF markers (ASPN, CXCL10, THY1) was detected only in CAFs but not in co-cultivated fibroblasts, which indicates the phenotypic difference between these cells. We assume that the in vitro activated normal fibroblasts in our study represent fibroblasts recently recruited to the tumor stroma rather than the resident CAFs of advanced tumors.

\subsection{Colorectal Cancer Cells, Normal Fibroblasts, and Their Co-Cultures Remodel Collagen in Different Modes}

The activation of fibroblasts normally occurs in response to tissue injury and inflammation. The main result of the activation is specific extracellular matrix remodeling: increased synthesis of ECM components such as collagen, fibronectin, elastin, and others, plus their deposition and remodeling [41]. Similar processes have been observed in tumors where they lead to a gradual stiffening of the tumor stroma due to increased collagen deposition and to an increased thickness, length, and alignment of the collagen fibers [13,14,18,55-59].

Stromal transformation is a complex process that encompasses a variety of chemical and physical mechanisms and processes. At least three main cell types participate in this phenomenon: cancer cells, CAFs, and tumor macrophages [60]. The function of CAFs is of the utmost importance and is under active consideration by many researchers [7,51,58]. Although the ability of cancer cells themselves to remodel the ECM has also been widely demonstrated, it has not yet been completely studied $[13,15,16,61,62]$. One of the few examples where the interactions of cancer cells with ECM were studied in detail is a series of publications that investigate the interactions of different types of breast cancer cells with fibronectin both in vitro and in vivo [63-65]. These studies show that more-mesenchymal breast cancer cells form the invasive front during tumor cell dissemination by fibronectin fibril alignment and that this alignment is transglutaminase- 2 mediated. At the same time, the secondary metastatic tumors consist of more-epithelial cancer cells with low fibronectin expression levels, and the survival of more-epithelial cells is more-mesenchymal cell-dependent [64]. It was also shown that transglutaminase 2 and fibronectin are capable of accumulating in extracellular vesicles [65] and that these vesicles induce fibroblast-mediated fibronectin fibril alignment [63].

In turn, our study has clearly demonstrated the ability of colorectal cancer cells with different epithelial/mesenchymal states to induce collagen alignment in vitro. Using the optical method of SHG microscopy, we showed both qualitatively and quantitatively that cancer cells with a mesenchymal 
phenotype (SW480) are able to form thick, oriented collagen fibers, unlike the non-invasive HT29 cell line. These findings are consistent with early observations that in prostate and breast cancers, the more invasive cancer cell lines show higher average fibril fractions and greater collagen compaction levels, with densely packed fibers when compared to non-invasive cell lines [62,66]. In addition, it was recently shown that mesenchymal breast cancer cells enhance the accumulation of fibronectin, the glycoprotein of the extracellular matrix, in the presence of activated fibroblasts. Moreover, the correlation of this process with the promotion of metastasis was demonstrated [63,64]. Our transcriptome analysis revealed that more genes related to collagen biosynthesis and positive regulation and to collagen catabolism and negative regulation are active in the SW480 cell line compared to HT29. Moreover, in spite of the fact that more genes related to collagen fibril organization and ECM aggregation were active in cell line HT29 compared to SW480, two important collagen-remodeling genes, LOXL2 and LOXL3 (lysyl oxidases), were more active in SW480s. The LOXL2 gene is considered as a promising target for cancer therapy [4]. The participation of LOXL2 in collagen maturation [67] and the ability of SW480 cells to remodel ECM, as demonstrated in our above experiments, make it possible to consider it as an important participant in the formation of the border collagen structure typical of cancers.

We simulated in vitro the recruitment of NFs to the neoplastic process and demonstrated that NFs and cancer cell cross-talk resulted in the establishment of a dense ECM formed by large highly oriented fibers. The collagen structure in the co-cultures differed from those in both corresponding cancer cell monocultures and CAFs. Notably, this structure is quite similar to those described for the tumor boundaries in ex vivo and in vivo studies $[13,18,60,68]$. For CAFs, the predominance of proteolytic processes leading to a decrease in collagen content but with the preservation of highly oriented large collagen fibers was demonstrated in vitro. All these data support the idea of a graduated development of the tumor stroma, as proposed recently by Emon et al. [60]. The authors suggest that at the first stages of tumor formation, cancer cells recruit and activate fibroblasts, which, in turn, facilitate EMT in cancer cells through growth factors and ECM stiffening. In the further stages, ECM degradation processes are activated in tumors that promote evasion and metastasis. Our transcriptomic data also support this hypothesis, since we observed the activation of collagen remodeling processes and the suppression of collagen catabolic processes in cancer cells, and the activation of collagen biosynthesis, collagen fibril organization, and ECM aggregation processes in fibroblasts as a result of co-cultivation. In addition, transcriptome analysis demonstrated an increased activity of collagen biosynthesis and organization genes in normal fibroblasts compared to CAFs, which could explain the more active ECM degradation by CAFs compared to NFs, since the expression of the collagen catabolism-related genes in these cells is similar. Thus, according to the characteristics of ECM remodeling, normal fibroblasts co-cultured with cancer cells are more consistent with recently recruited stromal cells, while patient-derived CAFs represent the stromal cells of advanced tumors.

The in vivo data obtained in our experiment are pilot and require further investigation. However, we have highlighted that the most invasive cell line with a mesenchymal phenotype, SW480, failed to initiate tumors in three cases out of four upon conventional s.c. injection into the thigh. Although this cell line is tumorigenic in mice, the tumors are characterized by an extremely slow growth and require specific conditions for inoculation (such as the use of Matrigel and/or localization in the anterior regions of the trunk) [69]. HT29, which showed a pronounced epidermal phenotype and low cell motility in vitro, induced the densest tumors, where the collagen fibers were larger and more highly oriented compared to those in HCT116 tumors. This could be related to the ability of HT29 cells to induce and support an activated state of recruited NFs, as was demonstrated in vitro. In turn, this leads to the formation of the specific ECM structure typical of co-cultivated NFs, which was also demonstrated in our results reported above. Previous reports have indicated the unexpected discrepancy between the low in vitro invasiveness and high in vivo invasiveness and metastatic activity of the HT29 line [70].

These observations and numerous data about the ECM-mediated promotion of the invasive and metastatic potential of cancer cells underlie the need for further investigations in this field 
in order to discover new therapeutic targets and drug candidates to interfere with the cancer cell-fibroblasts interplay.

\section{Materials and Methods}

\subsection{Cell Cultures}

Five human colorectal adenocarcinoma cell lines (HT29, HCT116, SW480, Caco-2, and SW837, all lines obtained from the Ivanovsky Institute of Virology, Moscow), human skin fibroblasts, and tumor-derived cancer-associated fibroblasts (CAFs) were used in the study. The colorectal human adenocarcinoma cell lines were routinely grown in Dulbecco's modified eagle's medium (DMEM; Gibco, Life Technologies, Carlsbad, CA, USA) supplemented with $10 \%$ fetal bovine serum FBS (HyClone, Logan, UT, USA), 2 mM glutamine (PanEco, Moscow, Russia), $10 \mathrm{mg} / \mathrm{mL}$ penicillin, and $10 \mathrm{mg} / \mathrm{mL}$ streptomycin at $37^{\circ} \mathrm{C}$ in a $5 \% \mathrm{CO}_{2}$ humidified atmosphere. The normal fibroblasts and CAFs were maintained in the same conditions as the cancer cells. Cells were routinely harvested at $80 \%$ confluence using $0.025 \%$ trypsin-EDTA (Gibco, Life Technologies, Carlsbad, CA, USA).

\subsection{Co-Culturing Cancer Cells and Fibroblasts in a Collagen-Based 3D Model}

Normal human fibroblasts (NFs) and colorectal cancer cell lines were used to develop a three-dimensional tumor model based on type I rat tail collagen. The collagen type I was derived from rat tails according to the standard protocol [71]. To obtain a three-dimensional collagen matrix model, we mixed 3.5 volumes of collagen solution $(1.5 \mathrm{mg} / \mathrm{mL})$ with one volume of a reagent mixture (10x Medium 199 (Gibco, Life Technologies, Carlsbad, CA, USA), NaOH, Na2CO3, glutamine and 1x 4-(2-hydroxyethyl)-1-piperazineethanesulfonic acid (HEPES)). For obtaining the co-culture, the tumor cells and fibroblasts were mixed in a ratio of 1:1.2. Then, a suspension of the cells was mixed with the collagen gel in the ratio of 1:10. The total cell concentration was $1 \times 10^{5}$ cells $/ \mathrm{mL}$ with a final concentration of collagen gel of $1.2 \mathrm{mg} / \mathrm{mL}$. Then, $500 \mu \mathrm{L}$ of the mixture was immediately pipetted and added to a $35 \mathrm{~mm}^{2}$ glass-bottom dish and incubated for $30 \mathrm{~min}$ at $37^{\circ} \mathrm{C}$. Then, $2 \mathrm{~mL}$ of culture medium were added and routinely changed once every two days. Monocultures of each cell line, including the CAFs, were cultured under the same conditions and used as controls. Observations were made on days $1,2,5$, and 7 of cultivation.

\subsection{Isolation of CAFs and Normal Fibroblasts}

The colon tumor samples were provided by the Volga Regional Medical Center (Nizhny Novgorod, Russia), while healthy skin samples were obtained from the Nizhny Novgorod Regional Children's Clinical Hospital in accordance with protocols approved by the local ethical committee (approval No. 6, 17 April 2019). The samples of tumor-associated fibroblasts (CAFs) from patient tumor samples and normal human skin fibroblasts from healthy skin were obtained using standard techniques with minor modifications [72]. Clinically, the tumor samples $(n=3)$ represented moderately differentiated colorectal adenocarcinomas, which were classified as stage III or IV (TMN) (Table S1). The tissue samples (tumor or skin) were repeatedly washed with culture medium with 10-fold antibiotic content until transparency of the supernatant was achieved. After that, the samples were crushed with sterile scissors. Then, enzymatic digestion was performed using Liberase ${ }^{\mathrm{TM}}$ (Roche, Belmont, CA, USA) as a dissociating agent in DMEM/F12 (Gibco, Life Technologies, Carlsbad, CA, USA) with the addition of hyaluronidase for $15 \mathrm{~min}$ at $37^{\circ} \mathrm{C}$ and constant stirring. Then, fresh medium was added for enzymes neutralization, and the suspension was centrifuged at $1.6 \times 10^{3} \mathrm{rpm}$ for $7 \mathrm{~min}$. The supernatant was removed, and the cell pellet with tissue pieces was resuspended in ACK Lysing Buffer (Gibco, Life Technologies, Carlsbad, CA, USA) according to the manufacture's protocol to remove any red blood cells. Then, the cells were extensively washed, resuspended, and centrifuged in fresh DMEM medium. Then, the cell suspension was placed into $25-\mathrm{cm}^{2}$ culture flasks. Tissue pieces were removed after fibroblast adhesion. 


\subsection{Immunofluorescence}

For immunofluorescence analysis, the monocultures and co-culturing cells were seeded on $35 \mathrm{~mm}^{2}$ glass-bottom dishes covered with type-1 rat tail collagen in a growth medium. After 5 days of culturing, the expression of EMT markers and fibroblast-activated proteins was assessed by an immunocytochemical method using antibodies to E-cadherin (ab15148, Abcam, Cambridge, MA, USA), vimentin (ab16700, Abcam, Cambridge, MA, USA), FAP (ab28244, Abcam, Cambridge, MA, USA), aSMA (ab5694, Abcam, Cambridge, MA, USA), EpCam (ab20160, Abcam, Cambridge, MA, USA), and secondary antibodies conjugated with a fluorescent label, either Fluorescein isothiocyanate (FITC; ab6825, Abcam, Cambridge, MA, USA) or Alexa (ab6825, Abcam, Cambridge, MA, USA). Staining was performed according to the antibody manufacturer's protocols, while the fluorescent dye DAPI was used for staining the nuclei. Fluorescence was recorded using a Leica DMIL fluorescence microscope (Leica, Wetzlar, Germany) equipped with filters: A4 UV BP 360/40 400 BP 470/40 for DAPI, CFP ET YFP ET (Ex: BP 500/20, Em: BP 535/30) for FITC and TX2 green BP 560/40 595 BP 645/75 for Alexa.

\subsection{Wound-Healing Assay}

Cancer cells were cultured for four days in six-well plates to confluence, and wounds were created with $10-\mu \mathrm{L}$ pipette tips. Next, the cells were incubated as described above, and bright-field microscopic images of the wounds were taken from five randomly selected fields at 0,48 , and $72 \mathrm{~h}$.

\subsection{Spheroid Formation}

To generate spheroids, cancer cells were seeded (100 cells per well) into a 96-well ultra-low attachment round-bottom plate (Corning, NY, USA). The plates were incubated $\left(37^{\circ} \mathrm{C}, 5 \% \mathrm{CO}_{2}\right)$ for 7 days. Culture medium was gently changed every three days. Spheroid formation was monitored with an inverted light microscope (Leica, Wetzlar, Germany).

\subsection{Tumor Xenografts}

The protocol for our animal experiments was approved by the Ethical Committee of the Privolzhsky Research Medical University (Russia). Experiments were performed on female, athymic, nude mice of 20-22 g body weight, purchased from the Pushchino animal nursery (Pushchino, Russian Federation). To generate tumors, HT29, HCT116, or SW480 cells were injected subcutaneously in the left flank at a dose of $5 \times 10^{6}$ cells in $200 \mu$ L PBS.

\subsection{Fluorescence-Activated Cell Sorting}

In order to separate co-cultured cancer cells and NFs, the NFs were stained, before co-culturing, with $2 \mu \mathrm{M}$ 5-Chloromethylfluorescein diacetate (CMFDA; Sigma-Aldrich, St. Louis, MO, USA) in serum-free medium for $20 \mathrm{~min}$; then, they were washed in complete medium and mixed with the cancer cells. After 5 days of co-culturing, the cells were detached with trypsin solution and counterstained with $10 \mu \mathrm{M}$ propidium iodide for 10-30 min to discriminate dead cells. Live NFs and cancer cells were sorted with a FACSAria III sorter (BD Biosciences, Franklin Lakes, NJ, USA) into $15 \mathrm{~mL}$ falcon tubes filled with $5 \mathrm{~mL}$ of complete medium. Aliquots (5\%-10\%) of sorted cells were re-analyzed to check for sorting purity and were found to be in the range $97.5 \%-99.8 \%$. After sorting, the cells were washed once with PBS and processed for RNA isolation.

\subsection{RNA Isolation and Sequencing}

At least 250,000 cells were collected by centrifugation and lysed using ExtractRNA (Evrogen, Moscow, Russia). The cell lysates were stored at $-70^{\circ} \mathrm{C}$ until RNA extraction. RNA was extracted with RNA Clean \& ConcentratorTM-5 (Zymo Research, Irvine, CA, USA). DNase I treatment was performed in-column manner during the clean-up. The quality of the extracted RNA was 
determined using TapeStation 2200 (Agilent Technologies, Santa Clara, CA, USA) with Agilent High Sensitivity RNA ScreenTape (Agilent Technologies). Since the RNA Integrity Number (RINe) was greater than 7 for all samples, poly(A) RNA isolation was performed with a NEBNext Poly(A) mRNA Magnetic Isolation Module (New England BioLabs, Ipswich, MA, USA). The NEBNext ${ }^{\circledR}$ Ultra II Directional RNA Library Prep Kit for Illumina ${ }^{\circledR}$ with Sample Purification Beads (New England BioLabs) and a NEBNext ${ }^{\circledR}$ Multiplex Oligos for Illumina (Index Primers Set 1-3) (New England BioLabs) were used for the preparation of single-indexed libraries. The preparation of the libraries was performed in technical triplicate for HT29, SW480, NF, HT29 ${ }^{\mathrm{NF}}, \mathrm{SW} 480^{\mathrm{NF}}, \mathrm{NF}^{\mathrm{HT} 29}$, and $\mathrm{NF}^{\mathrm{SW} 480}$. For CAF1 and CAF2, the preparation of the libraries was performed in technical duplicate. After the preparation, the individually indexed libraries were mixed in equimolar amounts; the final mixture was analyzed with TapeStation using Agilent High Sensitivity DNA ScreenTape (Agilent Technologies). The sequencing was performed with an Illumina NovaSeq 6000 System (Illumina, San Diego, CA, USA, SP-type, single-end reads, $100 \mathrm{bp}$ ).

The quality of raw reads was assessed using the MultiQC tool [73]. Since the quality of the reads in all samples was acceptable, trimming was not performed. The raw reads were mapped on the human reference genome (hg38) using HISAT2 (Galaxy Version 2.1.0+galaxy5; [74]). The featureCounts (Galaxy Version 1.6.4+galaxy1; [75]) was used for counting reads to GENCODE release 33 annotated genes [76]. Mitochondrial genes were removed prior to analysis. The obtained read counts were analyzed with DESeq2 (Galaxy Version 2.11.40.6+galaxy1 [77]) and also were converted into transcripts per kilobase million (TPM) values. Then, the TPM values were averaged over the replicates. The data discussed in this publication have been deposited in NCBI's Gene Expression Omnibus [78] and are accessible through GEO Series accession number GSE15534 (https:/www.ncbi.nlm.nih.gov/geo/query/ acc.cgi?acc=GSE155343).

\subsection{Second Harmonic Generation (SHG) Microscopy}

The visualization of fibrillar collagen and the quantitative assessment of its biophysical properties in vitro and in vivo was performed using second harmonic generation (SHG) microscopy, the established optical method based on the ability of centrosymmetric structures, such as collagen fibers, to reconvert two-photon excitation to form newly emitted photons with half of the wavelength [79].

For SHG and two-photon fluorescence microscopy in vitro, a multiphoton tomograph MPTflex (JenLab GmbH, Jena, Germany) equipped with a tunable 80 MHz, 200 fs Ti:Sa laser MaiTai (Spectra Physics, Santa Clara, CA, USA) was used. The objective lens was a Plan-Apochromat $40 \times / 1.3$ oil (Carl Zeiss, Oberkochen, Germany). The SHG signal from the collagen fibers was excited at a wavelength of $750 \mathrm{~nm}$ and detected in the range 373-387 $\mathrm{nm}$ in the backward direction. Two-photon fluorescence was excited at a wavelength of $750 \mathrm{~nm}$ and detected in the range $410-660 \mathrm{~nm}$. The average power applied to the sample was $\approx 12 \mathrm{~mW}$.

The in vivo SHG and two-photon fluorescence images were acquired using a LSM 880 laser scanning microscope (Carl Zeiss) equipped with a tunable $80 \mathrm{MHz}, 140 \mathrm{fs}$ Ti:Sa laser MaiTai HP (Spectra Physics). The images were acquired with a C Plan-Apochromat 40x/1.3 NA oil immersion objective through a mounted coverslip that was placed on the tumor surface. The SHG signal from the collagen fibers was excited at $800 \mathrm{~nm}$ and detected in the range $371-421 \mathrm{~nm}$ in the backward direction. Two-photon fluorescence was excited at a wavelength of $800 \mathrm{~nm}$ and detected in the range $433-660 \mathrm{~nm}$. The average power applied to the sample was $\approx 9 \mathrm{~mW}$.

SHG imaging was implemented in vivo on the 14-15th day of tumor growth. Before the imaging procedures, the mice were anesthetized intramuscularly with a mixture of Zoletil (40 mg/kg, $50 \mu \mathrm{L}$, Virbac SA, Carros, France) and 2\% Rometar (10 mg/kg, $10 \mu \mathrm{L}$, Spofa, Prague, Czech Republic), and a skin flap over the tumor was surgically opened. Once imaging was completed, the animals were sacrificed by cervical dislocation, and the tumors were excised for histopathological verification. 


\subsection{Quantitative Analysis of Collagen}

To evaluate the amount of collagen and its local organization, the following parameters of the SHG signal were calculated: the mean intensity, the density, the median, the non-uniformity, kurtosis, skewness, coherency, and energy [80].

The mean value is the simplest first-order statistical parameter used to evaluate the collagen SHG signal, and it correlates with the amount of collagen. The higher the mean value, the more collagen in the ROI. Density is also a characteristic of the amount of collagen in the SHG image. This parameter is normalized to the ROI area; therefore, it does not depend on the image size. The median parameter shows the most common value of the number of collagen fibers in the field of view. The non-uniformity parameter (S) is calculated as the ratio of the standard deviation to the mean value of the SHG signal. A higher $S$ value indicates a more heterogeneous distribution of the collagen. Skew (histogram asymmetry) is a third-order moment that determines the degree of symmetry of the gray distribution relative to the average. SHG images with thinner collagen fibers have higher asymmetry. Kurtosis is the fourth-order moment that defines the difference between the observed distribution in the histogram and a Gaussian distribution. The greater the value of the kurtosis parameter, the brighter the fibers. Energy (uniformity) shows the degree of variability of the SHG signal in the image. The higher the value, the more uniform the collagen structure. Coherence is a measure of orderliness or the presence of a dominant fiber direction. The larger the value, the more ordered the collagen fibers (there is a specific direction). The value of the coherency is in the range $0-1$, where 1 corresponds to a highly oriented structure, and 0 corresponds to isotropic areas. A detailed description of these quantitative metrics and their calculations can be found in [80].

Quantitative analysis of the SHG signal was performed using ImageJ software (National Institutes of Health, Bethesda, MD, USA) and the OrientationJ plugin. At each time point, 3-31 ROIs (each about $2500 \mathrm{\mu m}^{2}$ ) were assessed for each culture dish or tumor.

\subsection{Statistical Analysis}

The values are expressed as mean \pm standard deviation (SD) or the standard error of the mean (SEM). To estimate the statistical significance of the differences, the ANOVA with Bonferroni post-hoc test or a two-tailed Student's $t$-test were used where appropriate. The $p$-values $\leq 0.05$ were considered significant.

\section{Conclusions}

The role of the stroma in promoting cancer growth and metastasis is now a commonly accepted concept $[1,2,27]$. Normal fibroblasts recruited by cancer cells are necessary participants in the tumor invasion front; however, the molecular mechanisms of the cancer-stroma crosstalk are far from being well studied. In this report, we tried to correlate the dynamic processes of collagen biosynthesis and remodeling and the transcriptome changes during cancer and stroma cell interactions. To this end, we explored the impact of the change in the phenotype of colorectal cancer cells from epithelial to mesenchymal on their ability to activate fibroblasts and to remodel collagen structure in parallel with changes in the corresponding collagen-related gene expression.

Two data lines were identified:

Cancer cells impact on collagen structure and its change due to co-cultivation with fibroblasts

1. Cancer cells, when cultured alone, differ in their collagen remodeling capacity. An increase in the density of collagen was observed only for the SW480 (invasive) cell line, but not for HT29. SW480 demonstrated the ability to create quite thick, oriented fibers.

2. Co-culturing of colorectal cancer cell lines with normal fibroblasts resulted in a greater amount of fibrillar collagen during all periods of cultivation.

3. Fibroblast-mediated remodeling of collagen led to the formation of highly ordered fiber networks, which are more favorable for cancer cell invasion. However, after prolonged cultivation in the 
absence of cancer cells, the CAFs lost the ability to organize collagen in ways that promote invasion, and they actually initiated matrix degradation.

Expression of "collagen" genes in cancer cells and its change during co-cultivation with fibroblasts

1. The genes related to collagen biosynthesis and catabolism tend to be more active in the more-mesenchymal and mobile SW480 cells than in the more-epithelial and less mobile HT29 cells. Moreover, the LOXL2 and LOXL3 genes, which are necessary for collagen fibril organization, are SW480 specific.

2. The co-cultivation of colon cancer cell lines HT29 or SW480 with NFs leads to the activation of collagen biosynthesis and collagen fibril organization genes in both the fibroblasts and the cancer cells.

3. The genes related to collagen biosynthesis and collagen fibril organization tend to be more active in skin fibroblasts (NFs) than in colon CAFs, whereas CAF marker genes are significantly up-regulated in colorectal CAFs compared to normal skin fibroblasts.

4. The co-cultivation of the colorectal cancer cell lines HT29 or SW480 with skin fibroblasts leads to the activation of several CAF marker genes expression in the fibroblasts (ACTA2, POSTN, TNC, and DES). During co-cultivation, the HT29 line activates normal fibroblasts to a greater extent than does SW480.

Our findings suggest that normal fibroblasts, activated by cancer cells, strongly contribute to the organization of the extracellular matrix, thus being favorable for migratory potency. We also hope that further analysis of our transcriptomic data will help us find new therapeutical targets among the genes that mediate cancer cell-fibroblast interactions. In conclusion, targeting the ability of cancer cells to activate normal fibroblasts can be considered an important field in which to search for new therapeutic strategies.

Supplementary Materials: Supplementary Materials can be found at http://www.mdpi.com/1422-0067/21/21/ 8119/s1, Figure S1: Comparison of transcriptomes for HT29 and SW480 colorectal cancer cell lines and subsequent Gene Ontology-based functional enrichment analysis of DEGs, Figure S2: Expression of the genes, related to collagen metabolism and its regulation in normal fibroblasts and CAFs, isolated from the patient's colorectal tumors, Figure S3: Differential expression of the collagen-related genes in co-cultivated cancer cells and fibroblasts, Table S1: Clinicopathologic characteristics of surgical specimens used for CAFs isolation, Table S2. List of epithelial marker genes, Table S3: List of mesenchymal marker genes, Table S4: List of CAF marker genes, Table S5: Quantitative parameters of SHG signal in co-cultures of colorectal cancer cells and normal fibroblasts in a collagen-based model, Table S6: Quantitative parameters of SHG signal in monocultures of colorectal cancer cells in a collagen-based model, Table S7: Quantitative parameters of SHG signal in monocultures of normal fibroblasts (NF) and patient-derived CAFs in a collagen-based model, Table S8: List of ECM and collagen biosynthesis, collagen positive regulation, and COL genes (B+PR gene category), Table S9: List of collagen fibril organization and ECM aggregation related genes (CFO+EA gene category), Table S10: List of collagen catabolism and negative regulation related genes (C+NR gene category).

Author Contributions: Conceptualization, I.D., D.D. and E.S.; Data curation, D.D. and O.R.; Formal analysis, I.D., V.D., D.S., D.D. and O.R.; Funding acquisition, M.S., S.K. and E.S.; Investigation, N.I., M.L., D.S., D.D., A.K., G.S. and O.R.; Methodology, I.D., V.D., M.L. and D.D.; Project administration, M.S. and I.A.; Resources, M.S., S.K. and I.A.; Supervision, M.S., E.Z. and I.A.; Visualization, I.D., M.S., N.I., V.D., D.D., A.K. and O.R.; Writing-original draft, I.D., M.S., N.I., D.D., A.K., O.R., I.A. and E.S.; Writing-review and editing, M.S., E.Z., D.D., A.K., O.R., I.A. and E.S. All authors have read and agreed to the published version of the manuscript.

Funding: The reported study was funded by RFBR according to the research project No. 17-00-00194 (17-00-00190, 17-00-00189, 17-00-00193).

Conflicts of Interest: The authors declare no conflict of interest. The funders had no role in the design of the study; in the collection, analyses, or interpretation of data; in the writing of the manuscript, or in the decision to publish the results. 


\section{Abbreviations}

$\begin{array}{ll}\text { EMT } & \text { epithelial-mesenchymal transition } \\ \text { ECM } & \text { extracellular matrix } \\ \text { CAF } & \text { cancer-associated fibroblast } \\ \text { NF } & \text { normal fibroblast } \\ \text { SHG } & \text { second harmonic generation } \\ \text { IHC } & \text { immunohistochemistry } \\ \text { FACS } & \text { fluorescence-activated cell sorting } \\ \text { RNA-seq } & \text { RNA sequencing }\end{array}$

\section{References}

1. Hanahan, D.; Coussens, L.M. Accessories to the Crime: Functions of Cells Recruited to the Tumor Microenvironment. Cancer Cell 2012, 21, 309-322. [CrossRef] [PubMed]

2. Sverdlov, E. Missed Druggable Cancer Hallmark: Cancer-Stroma Symbiotic Crosstalk as Paradigm and Hypothesis for Cancer Therapy. Bioessays 2018, 40, 1800079. [CrossRef]

3. Valkenburg, K.C.; De Groot, A.E.; Pienta, K.J. Targeting the tumour stroma to improve cancer therapy. Nat. Rev. Clin. Oncol. 2018, 15, 366-381. [CrossRef] [PubMed]

4. Chopra, V.; Sangarappillai, R.M.; Romero-Canelón, I.; Jones, A.M. Lysyl Oxidase Like-2 (LOXL2): An Emerging Oncology Target. Adv. Ther. 2020, 3, 1900119. [CrossRef]

5. Sahai, E.; Astsaturov, I.; Cukierman, E.; DeNardo, D.G.; Egeblad, M.; Evans, R.M.; Fearon, D.; Greten, F.R.; Hingorani, S.R.; Hunter, T.; et al. A framework for advancing our understanding of cancer-associated fibroblasts. Nat. Rev. Cancer 2020, 20, 174-186. [CrossRef]

6. Nurmik, M.; Ullmann, P.; Rodriguez, F.; Haan, S.; Letellier, E. In search of definitions: Cancer-associated fibroblasts and their markers. Int. J. Cancer 2020, 146, 895-905. [CrossRef] [PubMed]

7. Nissen, N.I.; Karsdal, M.; Willumsen, N. Collagens and Cancer associated fibroblasts in the reactive stroma and its relation to Cancer biology. J. Exp. Clin. Cancer Res. 2019, 38, 115. [CrossRef] [PubMed]

8. Caporale, A.; Vestri, A.R.; Benvenuto, E.; Mariotti, M.; Cosenza, U.M.; Scarpini, M.; Giuliani, A.; Mingazzini, P.; Angelico, F. Is desmoplasia a protective factor for survival in patients with colorectal carcinoma? Clin. Gastroenterol. Hepatol. 2005, 3, 370-375. [CrossRef]

9. Sirica, A.E.; Gores, G.J. Desmoplastic stroma and cholangiocarcinoma: Clinical implications and therapeutic targeting. Hepatology 2014, 59, 2397-2402. [CrossRef]

10. Pickup, M.W.; Mouw, J.K.; Weaver, V.M. The extracellular matrix modulates the hallmarks of cancer. EMBO Rep. 2014, 15, 1243-1253. [CrossRef]

11. Walker, C.; Mojares, E.; del Río Hernández, A. Role of Extracellular Matrix in Development and Cancer Progression. Int. J. Mol. Sci. 2018, 19, 3028. [CrossRef] [PubMed]

12. Schwager, S.C.; Taufalele, P.V.; Reinhart-King, C.A. Cell-Cell Mechanical Communication in Cancer. Cell. Mol. Bioeng. 2019, 12, 1-14. [CrossRef] [PubMed]

13. Provenzano, P.P.; Inman, D.R.; Eliceiri, K.W.; Keely, P.J. Matrix density-induced mechanoregulation of breast cell phenotype, signaling and gene expression through a FAK-ERK linkage. Oncogene 2009, 28, 4326-4343. [CrossRef] [PubMed]

14. Acerbi, I.; Cassereau, L.; Dean, I.; Shi, Q.; Au, A.; Park, C.; Chen, Y.Y.; Liphardt, J.; Hwang, E.S.; Weaver, V.M. Human breast cancer invasion and aggression correlates with ECM stiffening and immune cell infiltration. Integr. Biol. (UK) 2015, 7, 1120-1134. [CrossRef] [PubMed]

15. Elia, I.; Rossi, M.; Stegen, S.; Broekaert, D.; Doglioni, G.; van Gorsel, M.; Boon, R.; Escalona-Noguero, C.; Torrekens, S.; Verfaillie, C.; et al. Breast cancer cells rely on environmental pyruvate to shape the metastatic niche. Nature 2019, 568, 117-121. [CrossRef] [PubMed]

16. Anguiano, M.; Morales, X.; Castilla, C.; Pena, A.R.; Ederra, C.; Martínez, M.; Ariz, M.; Esparza, M.; Amaveda, H.; Mora, M.; et al. The use of mixed collagen-Matrigel matrices of increasing complexity recapitulates the biphasic role of cell adhesion in cancer cell migration: ECM sensing, remodeling and forces at the leading edge of cancer invasion. PLOS ONE 2020, 15, e0220019. [CrossRef] 
17. Choi, S.Y.; Sung, R.; Lee, S.J.; Lee, T.G.; Kim, N.; Yoon, S.M.; Lee, E.J.; Chae, H.B.; Youn, S.J.; Park, S.M. Podoplanin, $\alpha$-smooth muscle actin or S100A4 expressing cancer-associated fibroblasts are associated with different prognosis in colorectal cancers. J. Korean Med. Sci. 2013, 28, 1293-1301. [CrossRef]

18. Wei, B.; Zhou, X.; Liang, C.; Zheng, X.; Lei, P.; Fang, J.; Han, X.; Wang, L.; Qi, C.; Wei, H. Human colorectal cancer progression correlates with LOX-induced ECM stiffening. Int. J. Biol. Sci. 2017, 13, 1450-1457. [CrossRef]

19. Stadler, M.; Scherzer, M.; Walter, S.; Holzner, S.; Pudelko, K.; Riedl, A.; Unger, C.; Kramer, N.; Weil, B.; Neesen, J.; et al. Exclusion from spheroid formation identifies loss of essential cell-cell adhesion molecules in colon cancer cells. Sci. Rep. 2018, 8, 1151. [CrossRef]

20. Janiszewska, M.; Primi, M.C.; Izard, T. Cell adhesion in cancer: Beyond the migration of single cells. J. Biol. Chem. 2020, 295, 2495-2505. [CrossRef]

21. Smyrek, I.; Mathew, B.; Fischer, S.C.; Lissek, S.M.; Becker, S.; Stelzer, E.H.K. E-cadherin, actin, microtubules and FAK dominate different spheroid formation phases and important elements of tissue integrity. Biol. Open 2019, 8, bio037051. [CrossRef] [PubMed]

22. Van Marck, V.L.; Bracke, M.E. Epithelial-Mesenchymal Transitions in Human Cancer. In Madame Curie Bioscience Database; Landes Bioscience: Austin, TX, USA, 2013. Available online: https://www.ncbi.nlm.nih. gov/books/NBK6362/.

23. Zhang, B.; Zhang, Z.; Xia, S.; Xing, C.; Ci, X.; Li, X.; Zhao, R.; Tian, S.; Ma, G.; Zhu, Z.; et al. KLF5 Activates MicroRNA 200 Transcription To Maintain Epithelial Characteristics and Prevent Induced Epithelial-Mesenchymal Transition in Epithelial Cells. Mol. Cell. Biol. 2013, 33, 4919-4935. [CrossRef] [PubMed]

24. Hyun, K.A.; Goo, K.B.; Han, H.; Sohn, J.; Choi, W.; Kim, S.I.; Jung, H.I.; Kim, Y.S. Epithelial-to-mesenchymal transition leads to loss of EpCAM and different physical properties in circulating tumor cells from metastatic breast cancer. Oncotarget 2016, 7, 24677-24687. [CrossRef]

25. Jędroszka, D.; Orzechowska, M.; Hamouz, R.; Górniak, K.; Bednarek, A.K. Markers of epithelial-tomesenchymal transition reflect tumor biology according to patient age and Gleason score in prostate cancer. PLoS ONE 2017, 12, e0188842. [CrossRef]

26. Orr, B.; Riddick, A.C.P.; Stewart, G.D.; Anderson, R.A.; Franco, O.E.; Hayward, S.W.; Thomson, A.A. Identification of stromally expressed molecules in the prostate by tag-profiling of cancer-associated fibroblasts, normal fibroblasts and fetal prostate. Oncogene 2012, 31, 1130-1142. [CrossRef] [PubMed]

27. Shiga, K.; Hara, M.; Nagasaki, T.; Sato, T.; Takahashi, H.; Takeyama, H. Cancer-associated fibroblasts: Their characteristics and their roles in tumor growth. Cancers 2015, 7, 2443-2458. [CrossRef] [PubMed]

28. Christodoulou, I.; Goulielmaki, M.; Devetzi, M.; Panagiotidis, M.; Koliakos, G.; Zoumpourlis, V. Mesenchymal stem cells in preclinical cancer cytotherapy: A systematic review. Stem Cell Res. Ther. 2018, 9, 1-38. [CrossRef]

29. Fukagawa, D.; Sugai, T.; Osakabe, M.; Suga, Y.; Nagasawa, T.; Itamochi, H.; Sugiyama, T. Protein expression patterns in cancer-associated fibroblasts and cells undergoing the epithelial-mesenchymal transition in ovarian cancers. Oncotarget 2018, 9, 27514-27524. [CrossRef]

30. Su, S.; Chen, J.; Yao, H.; Liu, J.; Yu, S.; Lao, L.; Wang, M.; Luo, M.; Xing, Y.; Chen, F.; et al. CD10+GPR77+ Cancer-Associated Fibroblasts Promote Cancer Formation and Chemoresistance by Sustaining Cancer Stemness. Cell 2018, 172, 841.e16-856.e16. [CrossRef]

31. Yeo, S.Y.; Lee, K.W.; Shin, D.; An, S.; Cho, K.H.; Kim, S.H. A positive feedback loop bi-stably activates fibroblasts. Nat. Commun. 2018, 9, 1-16. [CrossRef]

32. Wong, P.F.; Wei, W.; Gupta, S.; Smithy, J.W.; Zelterman, D.; Kluger, H.M.; Rimm, D.L. Multiplex quantitative analysis of cancer-associated fibroblasts and immunotherapy outcome in metastatic melanoma. J. Immunother. Cancer 2019, 7, 194. [CrossRef] [PubMed]

33. Curtis, M.; Kenny, H.A.; Ashcroft, B.; Mukherjee, A.; Johnson, A.; Zhang, Y.; Helou, Y.; Batlle, R.; Liu, X.; Gutierrez, N.; et al. Fibroblasts Mobilize Tumor Cell Glycogen to Promote Proliferation and Metastasis. Cell Metab. 2019, 29, 141.e9-155.e9. [CrossRef] [PubMed]

34. Pavón, M.A.; Arroyo-Solera, I.; Céspedes, M.V.; Casanova, I.; León, X.; Mangues, R. uPA/uPAR and SERPINE1 in head and neck cancer: Role in tumor resistance, metastasis, prognosis and therapy. Oncotarget 2016, 7, 57351-57366. [CrossRef]

35. Alekseenko, I.V.; Chernov, I.P.; Kostrov, S.V.; Sverdlov, E.D. Are synapse-like structures a possible way for crosstalk of cancer with its microenvironment? Cancers 2020, 12, 806. [CrossRef] 
36. Kalluri, R. The biology and function of fibroblasts in cancer. Nat. Rev. Cancer 2016, 16, 582-598. [CrossRef] [PubMed]

37. LeBleu, V.S.; Kalluri, R. A peek into cancer-associated fibroblasts: Origins, functions and translational impact. DMM Dis. Model. Mech. 2018, 11,1-18. [CrossRef]

38. Cortez, E.; Roswall, P.; Pietras, K. Functional subsets of mesenchymal cell types in the tumor microenvironment. Semin. Cancer Biol. 2014, 25, 3-9. [CrossRef]

39. Arina, A.; Idel, C.; Hyjek, E.M.; Alegre, M.L.; Wang, Y.; Bindokas, V.P.; Weichselbaum, R.R.; Schreiber, H. Tumor-associated fibroblasts predominantly come from local and not circulating precursors. Proc. Natl. Acad. Sci. USA 2016, 113, 7551-7556. [CrossRef]

40. Ishii, G.; Ochiai, A.; Neri, S. Phenotypic and functional heterogeneity of cancer-associated fibroblast within the tumor microenvironment. Adv. Drug Deliv. Rev. 2016, 99, 186-196. [CrossRef]

41. Darby, I.A.; Laverdet, B.; Bonté, F.; Desmoulière, A. Fibroblasts and myofibroblasts in wound healing. Clin. Cosmet. Investig. Dermatol. 2014, 7, 301-311.

42. Wessels, D.J.; Pradhan, N.; Park, Y.N.; Klepitsch, M.A.; Lusche, D.F.; Daniels, K.J.; Conway, K.D.; Voss, E.R.; Hegde, S.V.; Conway, T.P.; et al. Reciprocal signaling and direct physical interactions between fibroblasts and breast cancer cells in a 3D environment. PLoS ONE 2019, 14, e0218854. [CrossRef] [PubMed]

43. Koh, B.; Jeon, H.; Kim, D.; Kang, D.; Kim, K.R. Effect of fibroblast co-culture on the proliferation, viability and drug response of colon cancer cells. Oncol. Lett. 2019, 17, 2409-2417. [CrossRef] [PubMed]

44. Jeong, S.Y.; Lee, J.H.; Shin, Y.; Chung, S.; Kuh, H.J. Co-culture of tumor spheroids and fibroblasts in a collagen matrix-incorporated microfluidic chip mimics reciprocal activation in solid tumor microenvironment. PLoS ONE 2016, 11, e0159013. [CrossRef]

45. Foster, D.S.; Jones, R.E.; Ransom, R.C.; Longaker, M.T.; Norton, J.A. The evolving relationship of wound healing and tumor stroma. JCI Insight 2018, 3, e99911. [CrossRef]

46. Schnittert, J.; Bansal, R.; Storm, G.; Prakash, J. Integrins in wound healing, fibrosis and tumor stroma: High potential targets for therapeutics and drug delivery. Adv. Drug Deliv. Rev. 2018, 129, 37-53. [CrossRef] [PubMed]

47. Henriksson, M.L.; Edin, S.; Dahlin, A.M.; Oldenborg, P.A.; Öberg, Å.; Van Guelpen, B.; Rutegård, J.; Stenling, R.; Palmqvist, R. Colorectal cancer cells activate adjacent fibroblasts resulting in FGF1/FGFR3 signaling and increased invasion. Am. J. Pathol. 2011, 178, 1387-1394. [CrossRef]

48. Peng, C.; Zou, X.; Xia, W.; Gao, H.; Li, Z.; Liu, N.; Xu, Z.; Gao, C.; He, Z.; Niu, W.; et al. Integrin $\alpha$ v $\beta 6$ plays a bi-directional regulation role between colon cancer cells and cancer-associated fibroblasts. Biosci. Rep. 2018, 38, BSR20180243. [CrossRef]

49. Eberlein, C.; Rooney, C.; Ross, S.J.; Farren, M.; Weir, H.M.; Barry, S.T. E-Cadherin and EpCAM expression by NSCLC tumour cells associate with normal fibroblast activation through a pathway initiated by integrin $\alpha \mathrm{v} \beta 6$ and maintained through TGF $\beta$ signalling. Oncogene 2015, 34, 704-716. [CrossRef]

50. Gao, M.-Q.; Kim, B.G.; Kang, S.; Choi, Y.P.; Park, H.; Kang, K.S.; Cho, N.H. Stromal fibroblasts from the interface zone of human breast carcinomas induce an epithelial-mesenchymal transition-like state in breast cancer cells in vitro. J. Cell Sci. 2010, 123, 3507-3514. [CrossRef]

51. Liu, T.; Zhou, L.; Li, D.; Andl, T.; Zhang, Y. Cancer-Associated Fibroblasts Build and Secure the Tumor Microenvironment. Front. Cell Dev. Biol. 2019, 7, 60. [CrossRef]

52. Kubouchi, Y.; Yurugi, Y.; Wakahara, M.; Sakabe, T.; Haruki, T.; Nosaka, K.; Miwa, K.; Araki, K.; Taniguchi, Y.; Shiomi, T.; et al. Podoplanin expression in cancer-associated fibroblasts predicts unfavourable prognosis in patients with pathological stage IA lung adenocarcinoma. Histopathology 2018, 72, 490-499. [CrossRef] [PubMed]

53. Yurugi, Y.; Wakahara, M.; Matsuoka, Y.; Sakabe, T.; Kubouchi, Y.; Haruki, T.; Nosaka, K.; Miwa, K.; Araki, K.; Taniguchi, Y.; et al. Podoplanin expression in cancer-associated fibroblasts predicts poor prognosis in patients with squamous cell carcinoma of the lung. Anticancer Res. 2017, 37, 207-213. [CrossRef] [PubMed]

54. Shindo, K.; Aishima, S.; Ohuchida, K.; Fujiwara, K.; Fujino, M.; Mizuuchi, Y.; Hattori, M.; Mizumoto, K.; Tanaka, M.; Oda, Y. Podoplanin expression in cancer-associated fibroblasts enhances tumor progression of invasive ductal carcinoma of the pancreas. Mol. Cancer 2013, 12, 168. [CrossRef]

55. Levental, K.R.; Yu, H.; Kass, L.; Lakins, J.N.; Egeblad, M.; Erler, J.T.; Fong, S.F.T.; Csiszar, K.; Giaccia, A.; Weninger, W.; et al. Matrix Crosslinking Forces Tumor Progression by Enhancing Integrin Signaling. Cell 2009, 139, 891-906. [CrossRef] [PubMed] 
56. Mierke, C.T.; Sauer, F.; Grosser, S.; Puder, S.; Fischer, T.; Käs, J.A. The two faces of enhanced stroma: Stroma acts as a tumor promoter and a steric obstacle. NMR Biomed. 2018, 31, e3831. [CrossRef]

57. Malik, R.; Lelkes, P.I.; Cukierman, E. Biomechanical and biochemical remodeling of stromal extracellular matrix in cancer. Trends Biotechnol. 2015, 33, 230-236. [CrossRef]

58. Santi, A.; Kugeratski, F.G.; Zanivan, S. Cancer Associated Fibroblasts: The Architects of Stroma Remodeling. Proteomics 2018, 18, 1700167. [CrossRef]

59. Case, A.; Brisson, B.K.; Durham, A.C.; Rosen, S.; Monslow, J.; Buza, E.; Salah, P.; Gillem, J.; Ruthel, G.; Veluvolu, S.; et al. Identification of prognostic collagen signatures and potential therapeutic stromal targets in canine mammary gland carcinoma. PLoS ONE 2017, 12, e0180448. [CrossRef]

60. Emon, B.; Bauer, J.; Jain, Y.; Jung, B.; Saif, T. Biophysics of Tumor Microenvironment and Cancer MetastasisA Mini Review. Comput. Struct. Biotechnol. J. 2018, 16, 279-287. [CrossRef]

61. Sander, L.M. Modeling Contact Guidance and Invasion by Cancer Cells. Cancer Res. 2014, 74, 4588-4596. [CrossRef]

62. Chiu, C.-L.; Digman, M.A.; Gratton, E. Cell Matrix Remodeling Ability Shown by Image Spatial Correlation. J. Biophys. 2013, 2013, 1-8. [CrossRef]

63. Libring, S.; Shinde, A.; Chanda, M.K.; Nuru, M.; George, H.; Saleh, A.M.; Abdullah, A.; Kinzer-Ursem, T.L.; Calve, S.; Wendt, M.K.; et al. The dynamic relationship of breast cancer cells and fibroblasts in fibronectin accumulation at primary and metastatic tumor sites. Cancers 2020, 12, 1270. [CrossRef]

64. Jun, B.H.; Guo, T.; Libring, S.; Chanda, M.K.; Paez, J.S.; Shinde, A.; Wendt, M.K.; Vlachos, P.P.; Solorio, L. Fibronectin-expressing mesenchymal tumor cells promote breast cancer metastasis. Cancers 2020, 12, 2553. [CrossRef] [PubMed]

65. Shinde, A.; Paez, J.S.; Libring, S.; Hopkins, K.; Solorio, L.; Wendt, M.K. Transglutaminase-2 facilitates extracellular vesicle-mediated establishment of the metastatic niche. Oncogenesis 2020, 9, 1-12. [CrossRef] [PubMed]

66. Harjanto, D.; Maffei, J.S.; Zaman, M.H. Quantitative analysis of the effect of cancer invasiveness and collagen concentration on 3D matrix remodeling. PLoS ONE 2011, 6, e24891. [CrossRef] [PubMed]

67. Schmelzer, C.E.H.; Heinz, A.; Troilo, H.; Lockhart-Cairns, M.P.; Jowitt, T.A.; Marchand, M.F.; Bidault, L.; Bignon, M.; Hedtke, T.; Barret, A.; et al. Lysyl oxidase-like 2 (LOXL2)-mediated cross-linking of tropoelastin. FASEB J. 2019, 33, 5468-5481. [CrossRef]

68. Hanley, C.J.; Noble, F.; Ward, M.; Bullock, M.; Drifka, C.; Mellone, M.; Manousopoulou, A.; Johnston, H.E.; Hayden, A.; Thirdborough, S.; et al. A subset of myofibroblastic cancer-associated fibroblasts regulate collagen fiber elongation, which is prognostic in multiple cancers. Oncotarget 2016, 7, 6159-6174. [CrossRef]

69. Dipersio, L.P. Regional growth differences of human tumour xenografts in nude mice. Lab. Anim. 1981, 15, 179-180. [CrossRef] [PubMed]

70. De Both, N.J.; Vermey, M.; Dinjens, W.N.; Bosman, F.T. A comparative evaluation of various invasion assays testing colon carcinoma cell lines. Br. J. Cancer 1999, 81, 934-941. [CrossRef]

71. Rajan, N.; Habermehl, J.; Coté, M.-F.; Doillon, C.J.; Mantovani, D. Preparation of ready-to-use, storable and reconstituted type I collagen from rat tail tendon for tissue engineering applications. Nat. Protoc. 2006, 1, 2753-2758. [CrossRef]

72. Herrera, M.; Herrera, A.; Larriba, M.; Ferrer-Mayorga, G.; Herreros, A.; Bonilla, F.; Baulida, J.; Peña, C. Colon Cancer-associated Fibroblast Establishment and Culture Growth. Bio-protocol 2016, 6, e1773. [CrossRef]

73. Ewels, P.; Magnusson, M.; Lundin, S.; Käller, M. MultiQC: Summarize analysis results for multiple tools and samples in a single report. Bioinformatics 2016, 32, 3047-3048. [CrossRef] [PubMed]

74. Kim, D.; Langmead, B.; Salzberg, S.L. HISAT: A fast spliced aligner with low memory requirements. Nat. Methods 2015, 12, 357-360. [CrossRef]

75. Liao, Y.; Smyth, G.K.; Shi, W. featureCounts: An efficient general purpose program for assigning sequence reads to genomic features. Bioinformatics 2014, 30, 923-930. [CrossRef]

76. Frankish, A.; Diekhans, M.; Ferreira, A.-M.; Johnson, R.; Jungreis, I.; Loveland, J.; Mudge, J.M.; Sisu, C.; Wright, J.; Armstrong, J.; et al. GENCODE reference annotation for the human and mouse genomes. Nucleic Acids Res. 2019, 47, D766-D773. [CrossRef]

77. Love, M.I.; Huber, W.; Anders, S. Moderated estimation of fold change and dispersion for RNA-seq data with DESeq2. Genome Biol. 2014, 15, 550. [CrossRef] 
78. Edgar, R.; Domrachev, M.; Lash, A.E. Gene Expression Omnibus: NCBI gene expression and hybridization array data repository. Nucleic Acids Res. 2002, 30, 207-210. [CrossRef]

79. Campagnola, P.J.; Dong, C.-Y. Second harmonic generation microscopy: Principles and applications to disease diagnosis. Laser Photon. Rev. 2011, 5, 13-26. [CrossRef]

80. Dudenkova, V.V.; Shirmanova, M.V.; Lukina, M.M.; Feldshtein, F.I.; Virkin, A.; Zagainova, E.V. Examination of Collagen Structure and State by the Second Harmonic Generation Microscopy. Biochemistry 2019, 84, 89-107. [CrossRef]

Publisher's Note: MDPI stays neutral with regard to jurisdictional claims in published maps and institutional affiliations.

(C) 2020 by the authors. Licensee MDPI, Basel, Switzerland. This article is an open access article distributed under the terms and conditions of the Creative Commons Attribution (CC BY) license (http://creativecommons.org/licenses/by/4.0/). 